\title{
Feasibility Study of SDAS Instrumentation's Ability to Identify Mobile Launcher (ML)/Crawler-Transporter (CT) Modes during Rollout Operations
}

\author{
James P. Winkel, James C. Akers, NASA Glenn Research Center, Cleveland, OH 44095 \\ Erica Bruno, Analytical Mechanics Associates, Houston, TX 77058
}

\subsection{ABSTRACT}

The Space Launch System (SLS) and its Mobile Launcher (ML) will be transported to the launch pad via the CrawlerTransporter (CT) system. Rollout (i.e., transportation) loads produce structural loads on the integrated SLS/Orion MultiPurpose Crew Vehicle (MPCV) launch vehicle which are of a concern with respect to fatigue. As part of the risk reduction process and in addition to the modal building block test approach that has been adopted by the SLS Program, acceleration data will be obtained during rollout for use in modal parameter estimation. There are several occurrences where the ML/CT will be transported either into the Vertical Assembly Building (VAB) or to the launch pad and back without the SLS stack as part of the Kennedy Space Center (KSC) Exploration Ground Systems (EGS) Integrated Test and Checkout (ITCO). NASA KSC EGS has instrumentation installed on both the ML and CT to record data during rollout, at the launch pad, and during liftoff. The EGS instrumentation on the ML, which includes accelerometers, is referred to as the Sensor Data Acquisition System (SDAS). The EGS instrumentation on the CT, which also includes accelerometers, is referred to as the CT Data Acquisition System (CTDAS). The forces and accelerations applied to the ML and CT during a rollout event will be higher than any of the planned building block modal tests. This can be very beneficial in helping identify nonlinear behavior in the structure. Developing modal parameters from the same test hardware in multiple boundary conditions and under multiple levels of excitation is a key step in developing a well correlated FEM.

The purpose of this study was three fold. First, determine the target modes of the ML/CT in its rollout configuration. Second, determine if the test degrees of freedom (DOF) corresponding to the layout of the SDAS/CTDAS accelerometers (i.e. position and orientation) is sufficient to identify the target modes. Third, determine if the Generic Rollout Forcing Functions (GRFF's) [1] is sufficient for identifying the ML/CT target modes accounting for variations in CT speed, modal damping, and sensor/ambient background noise levels.

The finding from the first part of this study identified 28 target modes of the ML/CT rollout configuration based upon Modal Effective Mass Fractions (MEFF) and engineering judgement. The finding from the second part of this study showed that the SDAS/CTDAS accelerometers (i.e. position and orientation) are able to identify a sufficient number of the target modes to support model correlation of the ML/CT FEM. The finding from the third part of this study confirms the GRFFs sufficiently excite the ML/CT such that varying quantities of the defined target modes should be able to be extracted when utilizing an Experimental Modal Analysis (EMA) Multi-Input Multi-Output (MIMO) analysis approach. An EMA analysis approach was used because Operational Modal Analysis (OMA) tools were not available and the GRFFs were sufficiently uncorrelated. Two key findings from this third part of the study are that the CT speed does not show a significant impact on the ability to extract the modal parameters and that keeping the ambient background noise observed at each accelerometer location at or below $30 \mu \mathrm{grms}$ is essential to the success of this approach.

Even though this study relies heavily upon the accuracy of both uncorrelated ML and CT FEM's and unconfirmed rollout forcing functions, all of which will most likely differ from actuality, it provides important insights into the ability to ex tract modal parameters from the upcoming rollout events.

Keywords: Crawler Transporter, Launch Vehicle, Mobile Launcher, Space Launch System, Finite Element Model, Modal Damping, Modal Parameters, Sensor Noise, Target Modes, Time-Domain Force Response Analysis, Forcing Functions, Accelerometers, Rollout, NASA. 


\subsection{INTRODUCTION}

Major National Aeronautics and Space Administration (NASA) manned vehicles have made the final trip to the launch pad via a significant track/tread based system called the Crawler-Transporter (CT). Figure 1 depicts the Space Launch System (SLS) vehicle and the associated Mobile Launcher (ML) under transport to the launch site using this system. This "Rollout" event has been found to produce structural and fatigue loads on large flexible launch vehicles and spacecraft [2]. The dominant loading is due to the crossing of the truck rollers over the gaps between the track shoes. This produces two families of speed-dependent harmonics which coincide at the third and fifth harmonics producing significant loading amplification at those frequencies. The forces and accelerations applied to the ML and CT during a rollout event will be higher than any of the currently planned building block modal tests being carried out on the ML and CT by NASA Marshall Space Flight Center (MSFC). In an attempt to reduce the risks that a rollout event can pose to the SLS, acceleration data will be obtained during rollout for use in modal parameter estimation. Developing modal parameters from this higher excited rollout event will allow for further correlation of the ML and CT FEMs and ensure any nonlinear behavior of the structure is identified.

The purpose of this study is three fold. First, define the target modes of the ML/CT in its rollout configuration. Second, determine if the layout of the ML Sensor Data Acquisition System (SDAS)/CT Data Acquisition System (CTDAS) accelerometers (i.e. position and orientation) is sufficient to identify the target modes using the current uncorrelated Finite Element Models (FEM's). Third, determine if the Generic Rollout Forcing Functions (GRFF's) [Ref. 1] is sufficient for identifying the ML/CT target modes accounting for variations in CT speed, modal damping, and sensor/ambient background noise levels. Modal damping levels of 1\%,3\%, and 5\% and sensor/ambient background noise levels of $30 \mu \mathrm{grms}, 100 \mu \mathrm{grms}$, and $200 \mu \mathrm{grms}$ were looked at in this study. An Experimental Modal Analysis (EMA) Multi-Input Multi-Output (MIMO) analysis approach was used in this study because Operational Modal Analysis (OMA) tools were not available and the GRFFs were sufficiently uncorrelated. In Section 3.4, the correlation coefficients of the Generic Rollout Forcing Functions (GRFFs) were calculated per CT speed and verified the correlation between the GRFFs was sufficiently small at the specific speed ranges looked at in this study.

The findings and recommendations from this study are intended to inform engineers if the SDAS/CTDAS acceleration time histories from a rollout would provide sufficient modal parameters of the ML/CT for FEM correlation. If so, then rollouts occurring before before the ML Only modal test could be used to jump start ML/CT FEM correlation and rollouts occurring after the ML Only modal test can supplement the FEM correlation effort due to the higher excitation levels and the different boundary conditions occurring during rollout.

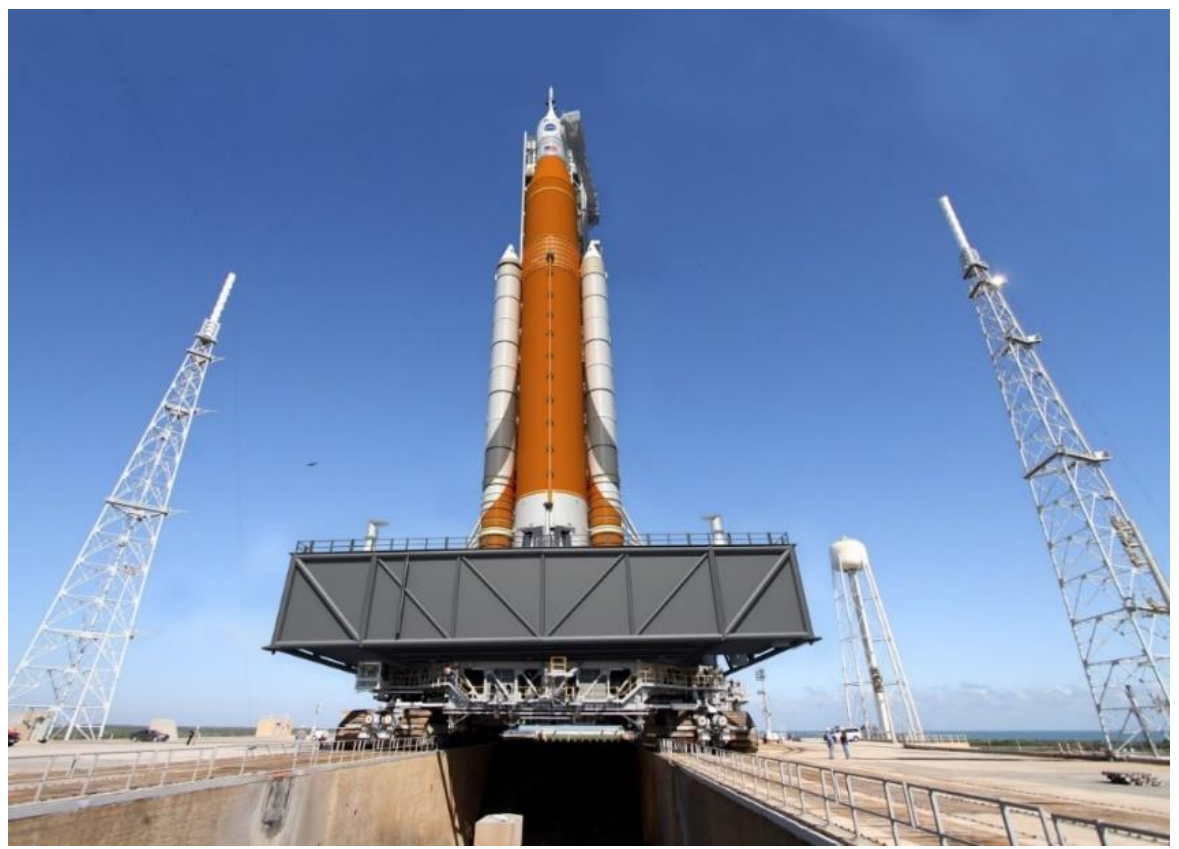

Figure 1. Artist Conception of the Space Launch System (SLS) and Mobile Launcher (ML) on the CrawlerTransporter (CT) (from http://www.nasa.gov) 


\subsection{ANALYSIS APPROACH}

\subsection{Hardware Configuration}

The ML and CT FEMs utilized in this study were provided via the NASA Johnson Space Center (JSC) team that also developed the GRFF's. The CT FEM is from the Space Shuttle era and includes lumped mass representations of the four trucks that drive the CT. The specific ML FEM used for this study is in the SLS rollout configuration. This means that it has the umbilicals and the Vehicle Stabilization System (VSS) in their deployed (extended) positions as it would be if the SLS was integrated onto the ML for transportation to the launch pad, and the Crew Access Arm (CAA) in its stowed configuration (i.e., retracted and rotated to lie along the $\mathrm{Y}+$ side of the ML Tower). Figure 2 shows the configured ML and CT FEM's used in this study. Figure 3 shows the layout of the ML, umbilicals, and CAA on the ML tower. Figure 4 shows the ML with the integrated SLS/Orion Multi-Purpose Crew Vehicle (MPCV) launch vehicle installed and the coordinate system utilized in the analysis. The X-direction is positive in the vertically downward orientation. The umbilicals are all attached on the Z+ side of the ML tower. The Crew Access Arm (CAA) is located on the Y+ side of the ML tower.

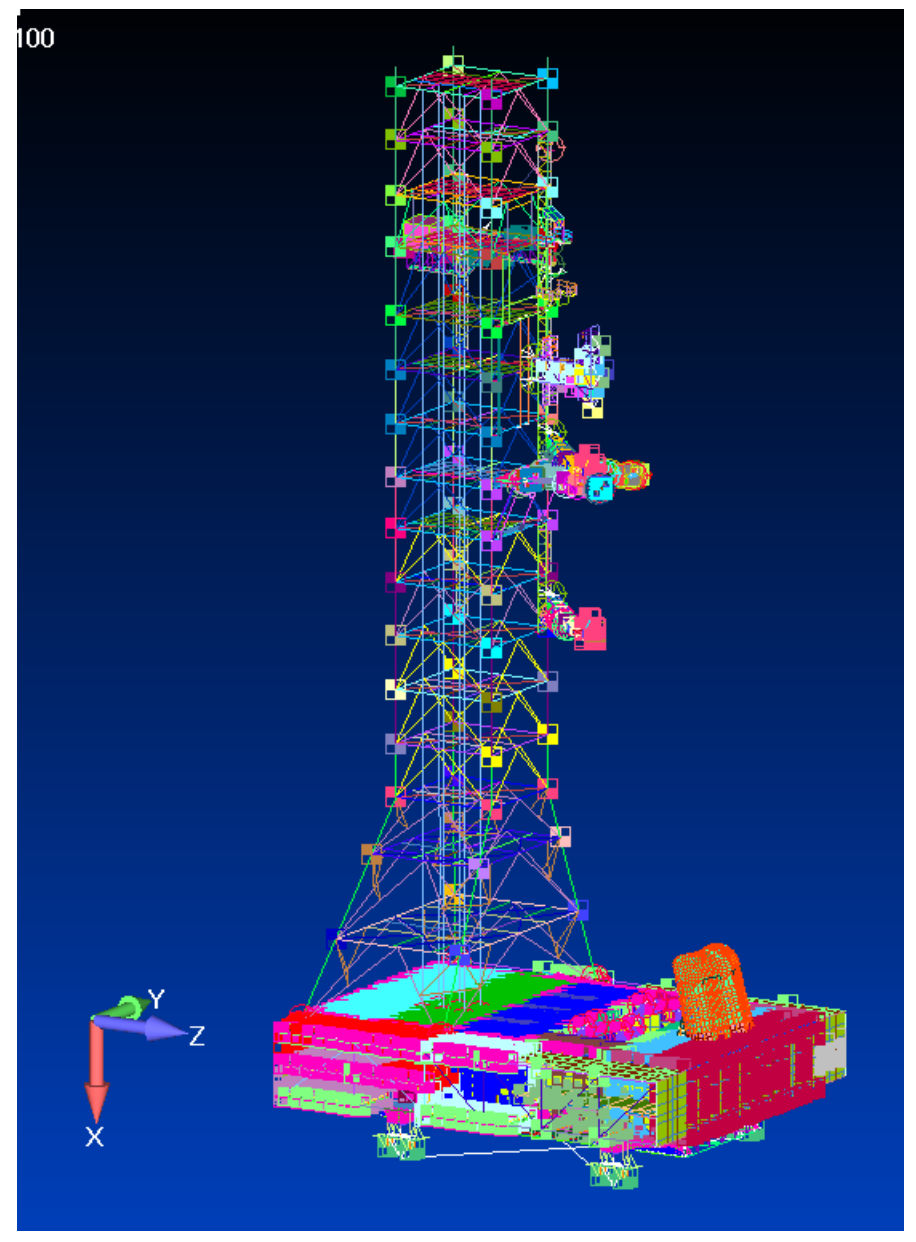

Figure 2. ML/CT FEM Configuration Utilized in Analytical Work

For all analysis done in the scope of this paper, the ML was in a unique configuration that is not considered a "normal" rollout configuration for the ML. For this analysis, the VSS is deployed (extended) as it would be if the SLS was integrated onto the ML for transportation to the launch pad. In addition, the Core Stage Intertank Umbilical (CSITU), Core State Forward Skirt Umbilical (CSFSU), and Interim Cryogenic Propulsion Stage Umbilical (ICPSU) are all in the deployed (extended) configurations. The Crew Access Arm (CAA) was in its stowed configuration. This rollout configuration is different from other planned rollout activities being performed, which will have all of the umbilicals and the VSS retracted. 

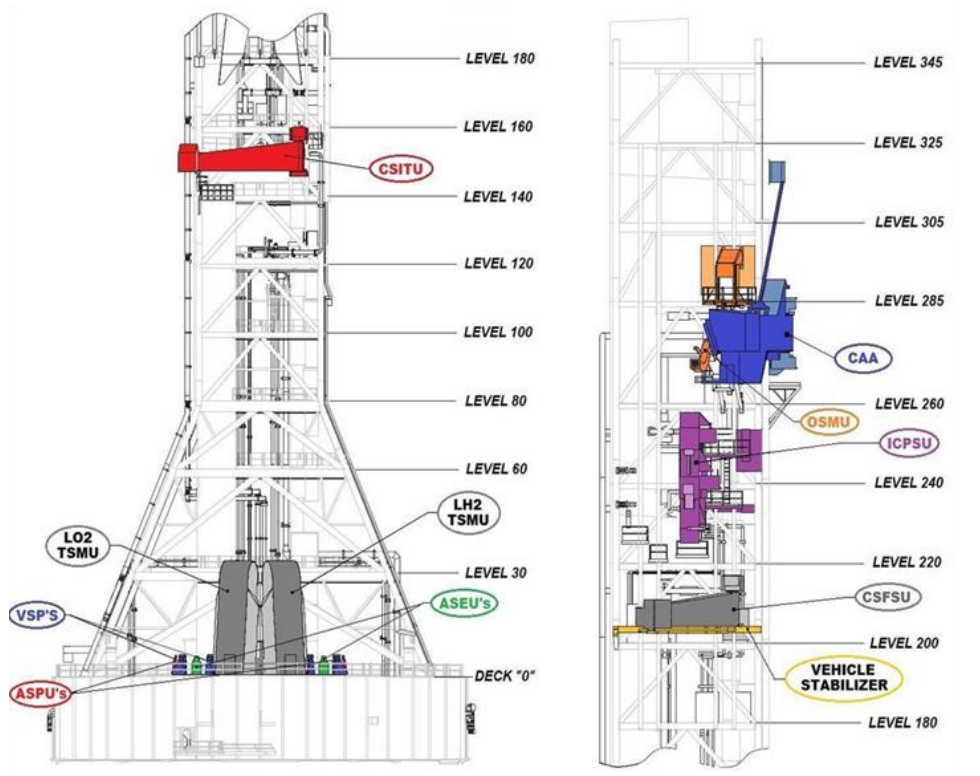

- Crew Access Arm (CAA)

- Orion Service Module Umbilical (OSMU)

- Interim Cryogenic Propulsion Stage Umbilical (ICPSU)

- Core Stage Intertank Umbilical (CSITU)

- Core Stage Forward Skirt Umbilical (CSFSU)

- (2) Aft Skirt Electrical Umbilical (ASEU) (2)

- (2) Aft Skirt Purge Umbilical (ASPU)

- Tail Service Mast Umbilical (TSMU) (LH2 \& LO2)

- Vehicle Stabilizer System (VSS)

- (8) Vehicle Support Posts (VSP)

Figure 3. ML, Umbilicals, and CAA Layout

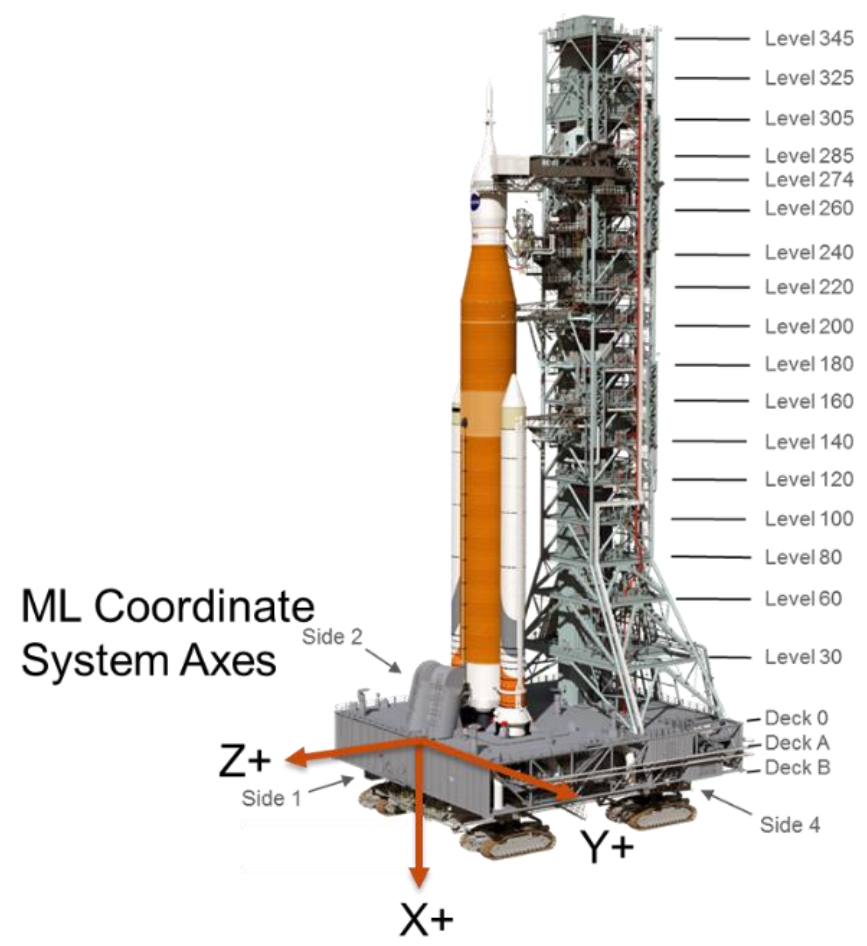

Figure 4. Schematic of the ML with the Integrated Launch Vehicle showing the ML Coordinate System

Figure 5 shows the CT, its vehicle coordinate system, and the labeling of its sides and corners. It should be pointed out that the boundary conditions are not the same at each of the four locations where the ML is attached to the CT. 


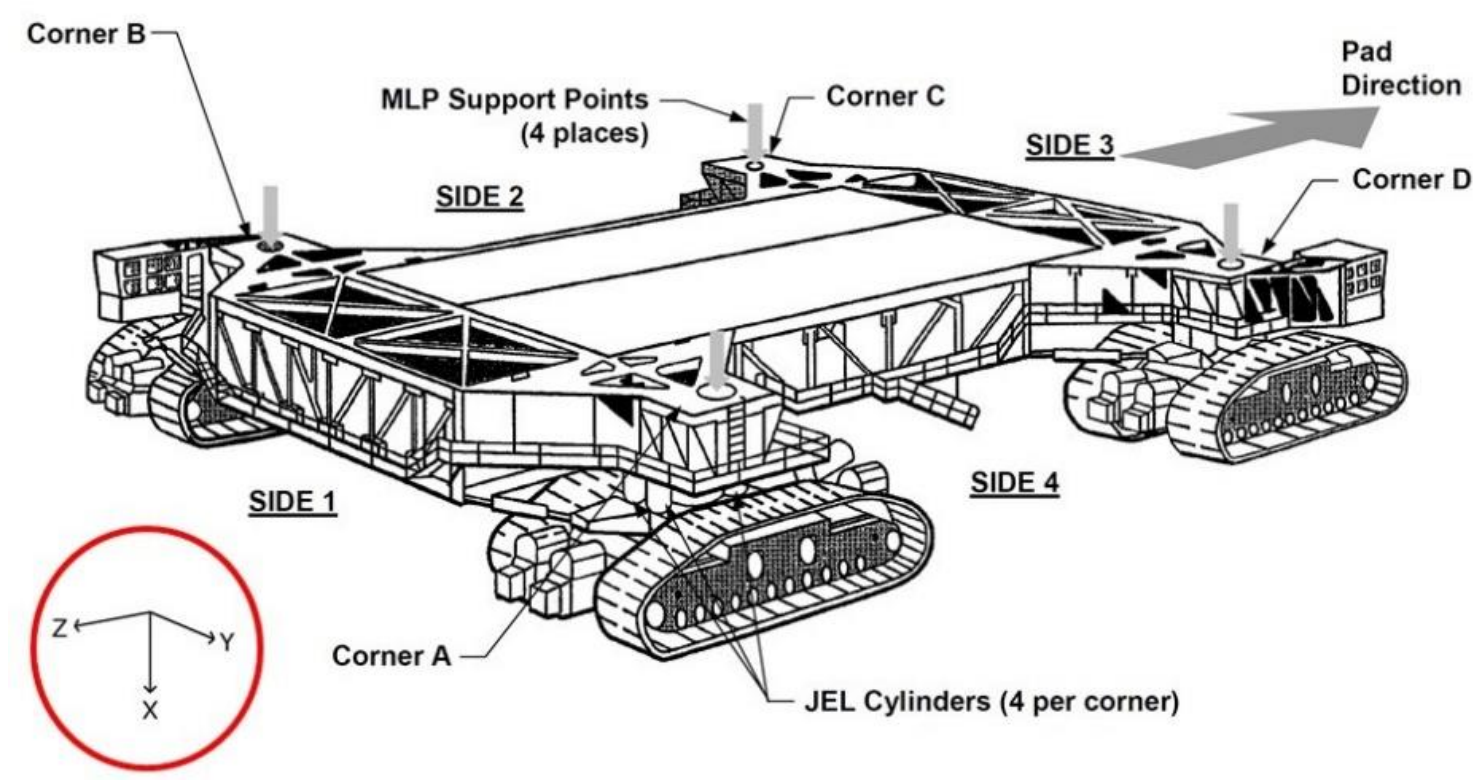

Figure 5. CT and its Vehicle Coordinate System.

The CT FEM used in this study has a highly simplified representation of the JEL system and trucks, shown in Figure 6 and Figure 7, compared to the actual hardware shown in Figure 7. The four CT ground interface points were constrained in all 6 degrees of freedom (DOF) by connecting them together with an RBE2 element branching out from a central grid point, which itself had all 6 DOF constrained. After collecting rollout data, this constraint should be evaluated to determine if it accurately represents the contact between the trucks and the ground during a rollout. In addition to this, all solid elements that were utilized in the ML and CT had their three rotational degrees of freedom (DOF) constrained using three separate single point constraint (SPC) sets.

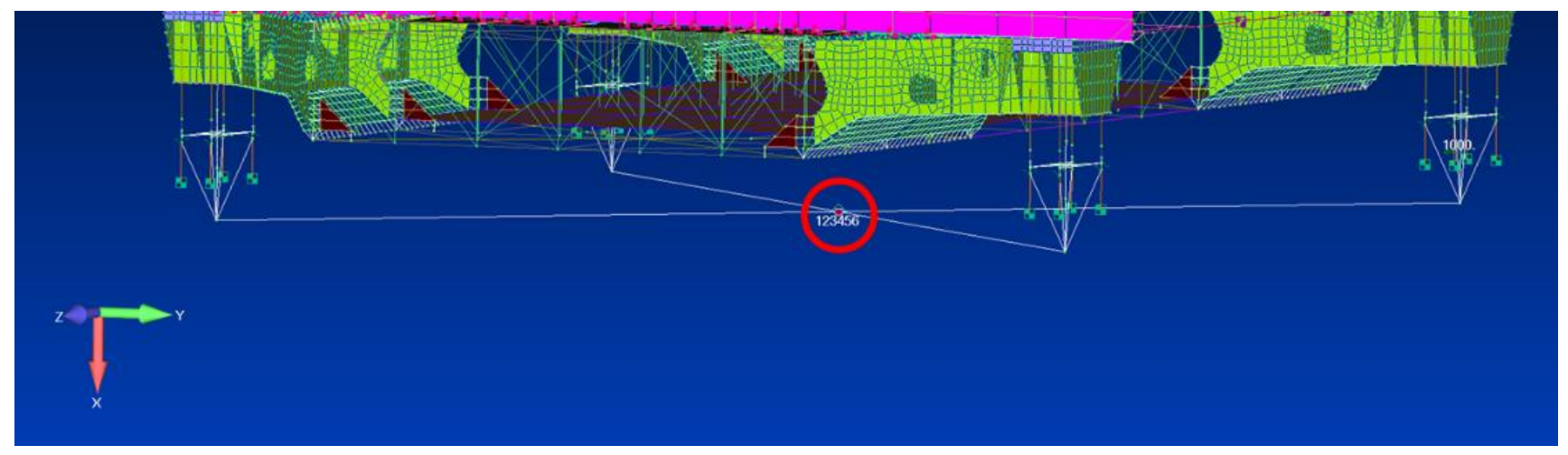

Figure 6. Fixed Central Grid Rigidly Attached to the Four CT Truck Ground Interface Locations

The RBE2 "Bowtie" shown in Figure 7 represents the top surface of the truck where the Jacking, Elevation, and Leveling (JEL) cylinders and guide tube connect into it. It is meant to identify the lateral stiffness created by that interface. The exact location of the below JEL accelerometer is indicated by the "RED" dot in Figure 7. The four light blue vertical beam elements in Figure 7 are the representation of each of the four JEL cylinders. These JEL cylinders are used to raise the CT chassis, to lift the ML or ML/SLS, and to keep the assembly level during rollout, including during transit up/down the ramp to the launch pad. 


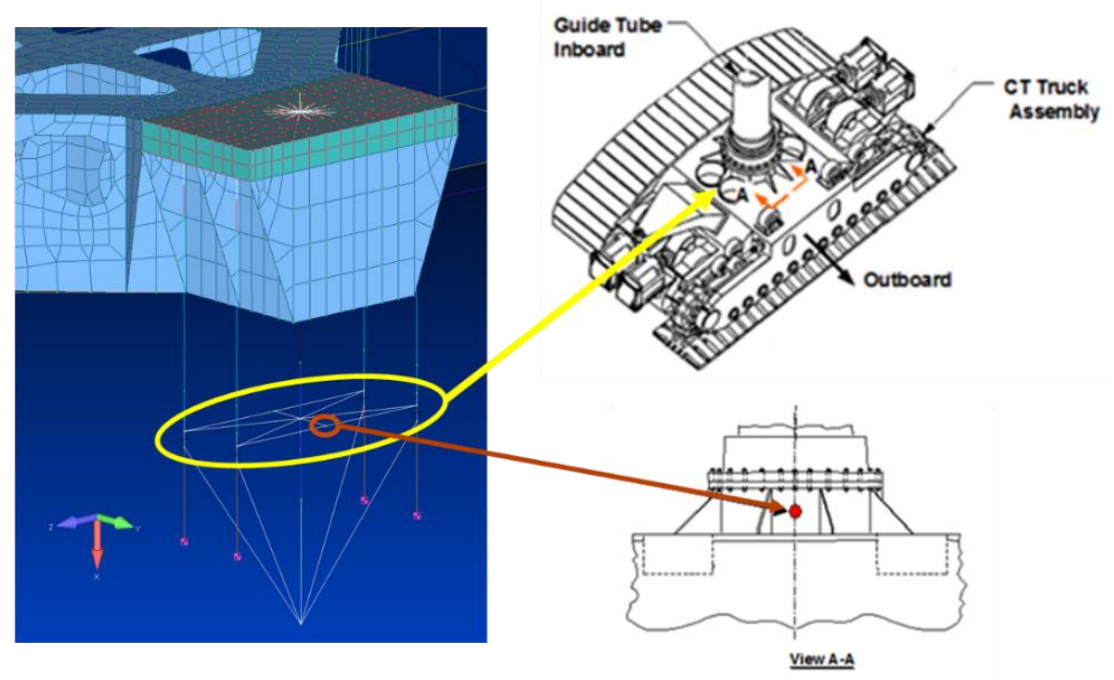

Figure 7. FEM vs. Hardware Relationship of the CT Truck

At the start of this study, some confusion existed as to what parts of the FEM represented which sections of the CT Truck hardware. This led to the selection of an incorrect location for the below JEL accelerometer location, which is also where the GRFFs were applied to the CT. Figure 8 shows the incorrect grid point selected for this study on the left and the correct grid point on the right. Unfortunately, this error was not caught until well after all the analytical work for this study had been completed. Fortunately, it was found that this error did not significantly impact the results of this study.
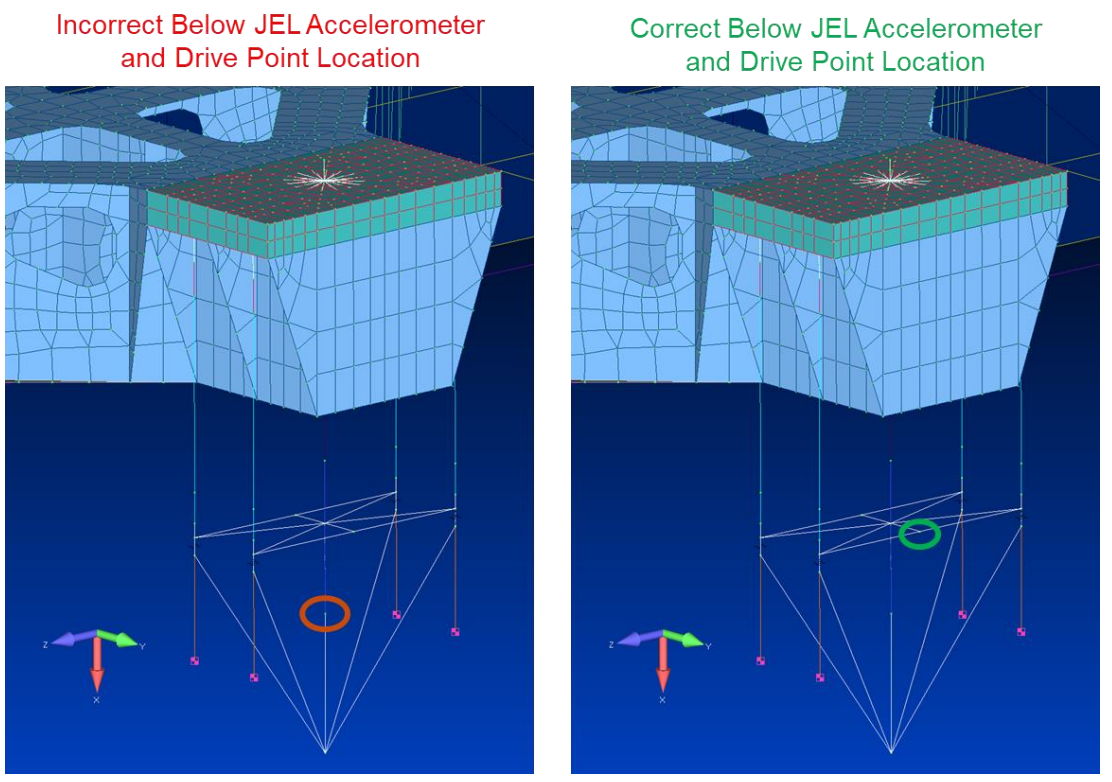

Figure 8. Truck Force FEM Application Point - Point Used in the Analysis (Left), Correct Point (Right)

There are four Multi-Point Constraint (MPC) elements which rigidly constrain the four pickup points of the CT to the ML in the vertical (X-Direction). Constraining the two lateral directions at each pickup point was done using CELAS2 spring elements. Each pickup of the CT has a different lateral stiffness modeled due to the difference in the physical connections that exist between the CT and ML. A different stiffness value was utilized if that direction was physically pinned versus being reliant on friction to keep the two sides of the joint from moving relative to one another. 
In order to accurately simulate the excitation that would be present in the ML/CT stack when it is in motion, the engineering team requires the actual forces generated at the base of the CT. However, this test data does not yet exist for either the SLS or rollout versions of the CT/ML stack. There is heritage test data from when the Space Shuttle mobile launch platform (MLP) was transported on the CT, but the SLS configuration of the ML/CT is considerably different than the Space Shuttle configuration. To address this issue, a team of engineers at NASA JSC developed an analytical methodology that could compute simulated forcing functions that would occur at the base of the CT during a rollout. The derivation of this approach is outside of the scope of this paper, but it is sufficiently documented in Reference 1. For this analysis effort, the GRFFs are applied to an SLS, ML and CT coupled system to recover accelerations above and below the JEL cylinders on the CT. These JEL cylinders are used to raise the CT chassis, to lift the ML, and to keep it level during rollout, including up the ramp to the launch pad. The CT had limited accelerometers on previous rollouts with triaxial accelerometers located above and below the JELs at each of the four pickup points and one at the lower center of the chassis. The interface between the CT and ML is defined just above each JEL. The rollout forcing functions developed by this process consist of three orthogonal translational forces below each of the four CT JELs for five speeds below $1 \mathrm{MPH}$. The three orthognal moments at each CT truck were assumed to be zero. The CT pickup points are intentionally designed not to transmit moments about any horizontal axis into the ML by incorporation of a half-spherical bearing (Figure 9).

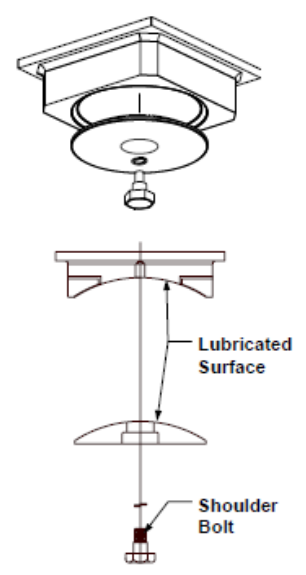

Figure 9. ML Pickup Point Mounting Block - Half-Spherical Bearing for Moment Removal [3]

\subsection{Target Modes}

Normal modes were computed in McNeil Schwindler (MSC) NASTRAN to a frequency range double the target frequency range of the rollout. As a best practice, modes were calculated to at least double the frequency range of interest to see if important modes might be slightly outside this frequency range. The modal results computed were compared back to the previously delivered modal results as a quick check of the FEM. They matched identically so no further FEM checkouts were made. For future analyses efforts, the standard FEM checkouts (e.g. free-free modes, $1 \mathrm{~g}$ loading in all three directions, enforced displacement, mass and cg checks, etc.,) should be performed.

The analytical results showed a modal density of $\sim 400$ modes up to double the target frequency range. Engineering best practice is to include a sufficient number of target modes to achieve $90 \%$ MEM in all 6 DOF. In this case, that guideline was relaxed slightly due to further analysis of the modes above the target frequency range and the fact that a significant amount of the CT mass is in the trucks, which have been grounded. Based upon the Modal Effective Mass Fractions (MEFF) metric, the first sequential 28 modes were identified as being the target modes for this study even if a few had very low MEFF. In this analytical study, it was decided after review of the mode shapes to keep a sequential set of modes in the range and not leave out any specific modes. This was purely done for convenience of the analysis and would not have carried onto the test. Using a summation of the MEFF in both the translational and rotational DOF, it is shown in Figure 10, that considering modes above the $28^{\text {th }}$ mode has dimensioning returns. 


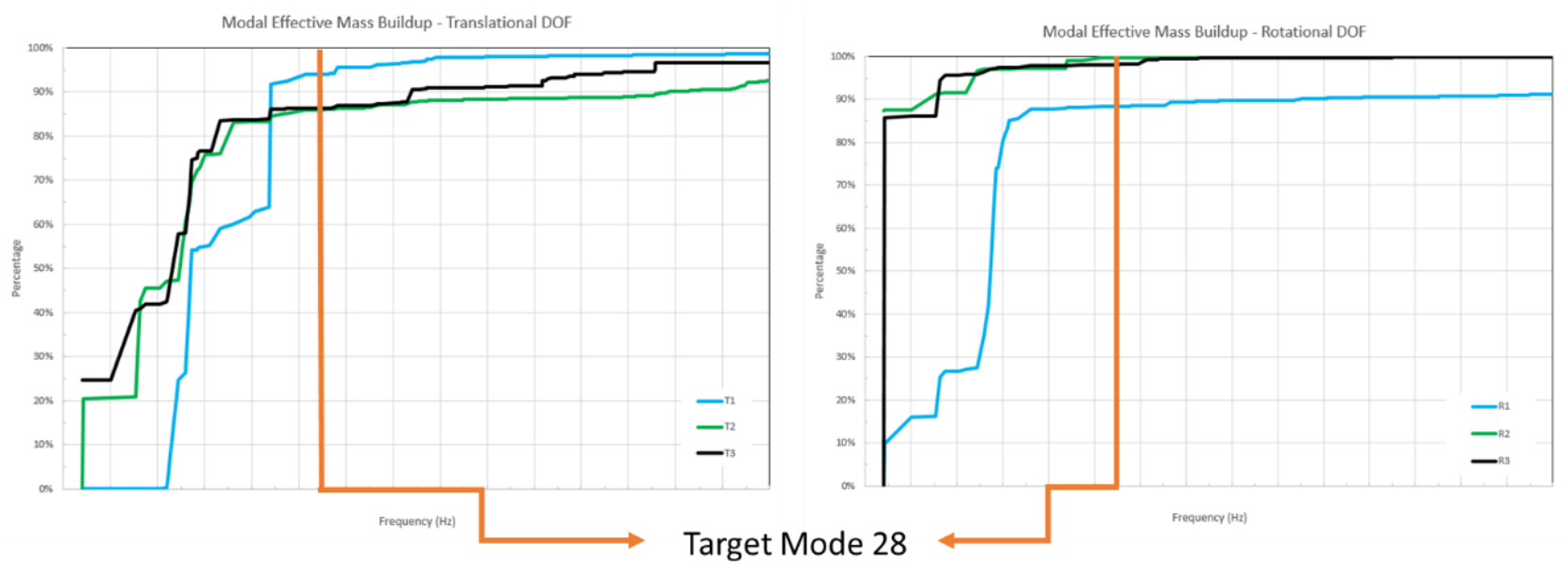

Figure 10. MEFF Summation

\begin{tabular}{|c|c|c|c|c|c|c|}
\hline Order & $\mathbf{T 1}$ & $\mathbf{T 2}$ & $\mathbf{T 3}$ & $\mathbf{R 1}$ & $\mathbf{R 2}$ & $\mathbf{R 3}$ \\
\hline 1 & $0.0 \%$ & $0.0 \%$ & $24.6 \%$ & $0.0 \%$ & $87.4 \%$ & $0.0 \%$ \\
\hline 2 & $0.0 \%$ & $20.5 \%$ & $0.0 \%$ & $9.8 \%$ & $0.0 \%$ & $85.8 \%$ \\
\hline 3 & $0.0 \%$ & $0.1 \%$ & $0.0 \%$ & $6.2 \%$ & $0.0 \%$ & $0.2 \%$ \\
\hline 4 & $0.0 \%$ & $0.3 \%$ & $15.9 \%$ & $0.1 \%$ & $3.8 \%$ & $0.1 \%$ \\
\hline 5 & $0.1 \%$ & $21.6 \%$ & $0.4 \%$ & $9.1 \%$ & $0.1 \%$ & $8.4 \%$ \\
\hline 6 & $0.0 \%$ & $3.1 \%$ & $0.9 \%$ & $1.5 \%$ & $0.2 \%$ & $1.0 \%$ \\
\hline 7 & $0.0 \%$ & $0.0 \%$ & $0.1 \%$ & $0.0 \%$ & $0.0 \%$ & $0.0 \%$ \\
\hline 8 & $0.1 \%$ & $1.6 \%$ & $0.5 \%$ & $0.4 \%$ & $0.1 \%$ & $0.3 \%$ \\
\hline & & & & & & \\
\hline 9 & $24.5 \%$ & $0.2 \%$ & $15.4 \%$ & $0.3 \%$ & $5.0 \%$ & $0.0 \%$ \\
\hline 10 & $1.5 \%$ & $13.0 \%$ & $0.3 \%$ & $7.8 \%$ & $0.3 \%$ & $0.5 \%$ \\
\hline 11 & $19.5 \%$ & $5.1 \%$ & $9.2 \%$ & $6.8 \%$ & $0.0 \%$ & $0.5 \%$ \\
\hline 12 & $8.5 \%$ & $4.0 \%$ & $7.3 \%$ & $6.6 \%$ & $0.0 \%$ & $0.2 \%$ \\
\hline 13 & $0.0 \%$ & $2.7 \%$ & $0.3 \%$ & $22.9 \%$ & $0.0 \%$ & $0.3 \%$ \\
\hline 14 & $0.4 \%$ & $0.2 \%$ & $1.2 \%$ & $2.3 \%$ & $0.0 \%$ & $0.0 \%$ \\
\hline 15 & $0.2 \%$ & $0.4 \%$ & $0.4 \%$ & $0.1 \%$ & $0.1 \%$ & $0.0 \%$ \\
\hline 16 & $0.1 \%$ & $3.0 \%$ & $0.0 \%$ & $6.7 \%$ & $0.0 \%$ & $0.1 \%$ \\
\hline 17 & $0.2 \%$ & $0.0 \%$ & $0.1 \%$ & $2.4 \%$ & $0.0 \%$ & $0.0 \%$ \\
\hline 18 & $0.6 \%$ & $0.0 \%$ & $0.0 \%$ & $2.0 \%$ & $0.0 \%$ & $0.0 \%$ \\
\hline 19 & $3.3 \%$ & $0.1 \%$ & $6.9 \%$ & $0.4 \%$ & $0.0 \%$ & $0.0 \%$ \\
\hline 20 & $1.2 \%$ & $7.2 \%$ & $0.1 \%$ & $2.2 \%$ & $0.0 \%$ & $0.4 \%$ \\
\hline 21 & $1.4 \%$ & $0.0 \%$ & $0.1 \%$ & $0.0 \%$ & $0.0 \%$ & $0.0 \%$ \\
\hline 22 & $1.4 \%$ & $0.0 \%$ & $0.0 \%$ & $0.0 \%$ & $0.0 \%$ & $0.0 \%$ \\
\hline 23 & $1.0 \%$ & $0.0 \%$ & $0.2 \%$ & $0.3 \%$ & $0.1 \%$ & $0.0 \%$ \\
\hline 24 & $27.9 \%$ & $1.3 \%$ & $2.2 \%$ & $0.1 \%$ & $1.6 \%$ & $0.0 \%$ \\
\hline 25 & $0.6 \%$ & $0.6 \%$ & $0.0 \%$ & $0.0 \%$ & $0.0 \%$ & $0.2 \%$ \\
\hline 26 & $0.0 \%$ & $0.0 \%$ & $0.2 \%$ & $0.0 \%$ & $0.1 \%$ & $0.0 \%$ \\
\hline 27 & $1.7 \%$ & $0.8 \%$ & $0.0 \%$ & $0.2 \%$ & $0.6 \%$ & $0.0 \%$ \\
\hline 28 & $0.0 \%$ & $0.0 \%$ & $0.0 \%$ & $0.0 \%$ & $0.0 \%$ & $0.0 \%$ \\
\hline Potal Mass & $\mathbf{9 4 . 1} \%$ & $\mathbf{8 6 . 0} \%$ & $\mathbf{8 6 . 4} \%$ & $\mathbf{8 8 . 4} \%$ & $\mathbf{9 9 . 7 \%}$ & $\mathbf{9 8 . 1} \%$ \\
\hline
\end{tabular}

Figure 11. Final Selected Target Mode Set for Analysis

\subsection{Instrumentation Pretest Analysis}

After the target mode set was selected, the next step was to determine if the layout of the SDAS/CTDAS accelerometers (i.e. position and orientation) was sufficient to identify the target modes. Figure 12 shows the cross-orthogonality and frequency comparison that was obtained by using the SDAS/CTDAS accelerometer set.

The cross-orthogonality between the Test Analysis Model (TAM) mode shapes and the analytical FEM mode shapes was used to evaluate the ability of the instrumentation set to identify a set of target modes. The TAM modes are the modes computed from the analytical FEM having undergone a Guyan (i.e. static) reduction to the SDAS/CTDAS set. A crossorthogonality matrix having 1.0 on the diagonal and zeros on the off-diagonals would indicate the TAM and FEM mode 
shapes exactly match with respect to the Guyan reduced mass matrix. In addition to the cross-orthogonality metric, a frequency comparison between similar modes is also provided. NASA provides standard guidelines for both crossorthogonality and frequency comparison results in NASA-STD-5002 [4]. For the cross-orthogonality, there should be greater than $90 \%$ values on the diagonal and less than $10 \%$ on off-diagonal. The difference in frequency should be less than $5 \%$ for correctly matched mode shapes.

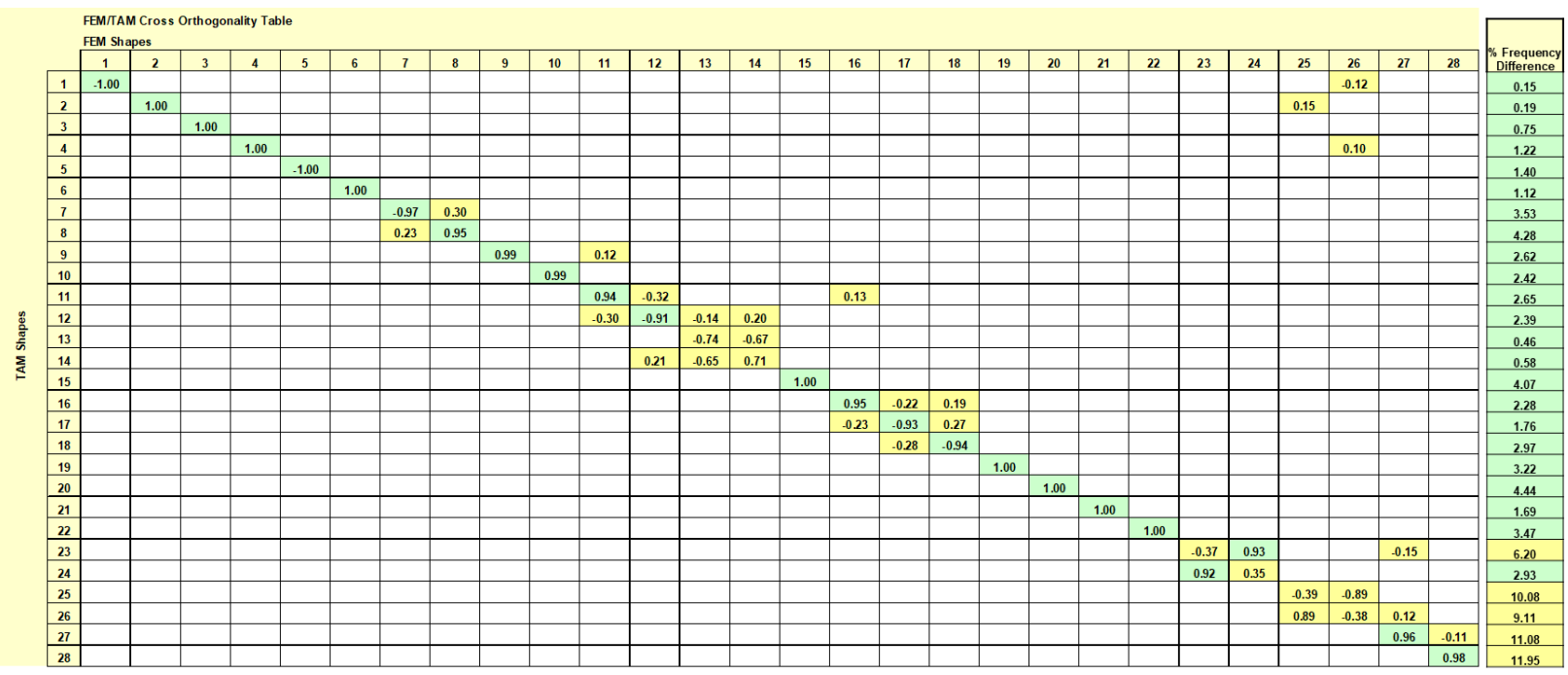

Figure 12. Pretest Instrumentation Metric for SDAS/CTDAS Accelerometer Set

The SDAS/CTDAS accelerometer set does not meet the NASA guidelines and would require additional DOF to improve. However, the single most important indicator in determining if an instrumentation set is "good enough" is ensuring the frequency comparison percentage is below 5\%. If it is above 5\%, there is a good chance the mode is missing a key DOF location. Second, mode pairs in large scale test articles always make it difficult to meet the NASA cross-orthogonality guideline because there is rarely enough instrumentation placed within close proximity of each other to differentiate the subtle differences between the two shapes. This will manifest itself in the cross-orthogonality matrix as four touching squares each having a value near $75 \%$. As the number of DOF increases that helps separate the two modes, the off-diagonal values will drop and the diagonal values will increase. Mode pairs are present for Modes 11-12, 13-14, and 17-18. Therefore, while the cross-orthogonality guidelines in NASA-STD-5002 are not met, based upon experience, the SDAS/CTDAS accelerometer set is adequate to identify the target modes up to Mode 23. In the next phase of the analytical effort, the goal is to determine if the GRFFs provided sufficent excition to identify this subset of target modes

\subsection{Force Response Analysis Data Quality Checks}

As a reminder, the point of the first step was to understand if the limited set of instrumentation present on the ML and CT during a rollout without the SLS was able to identify the target modes sufficently to start correlation of the FEM. The results obtained with the SDAS/CTDAS accelerometer set should allow for most of the target mode shapes to be extracted assuming the FEM and test article behave similarly. In the next step, the goal is to drive the analytical model at the CT trucks using forcing functions. This analysis is simulating an EMA MIMO modal extraction approach. Another valid approach would be Operational Modal Analysis (OMA), however, that approach is not considered in this paper. For an EMA approach, the recovery data would be time domain accelerations at all the instrumentation locations on the ML and CT. This would then be processed using standard test data processing tools as if the time domain data had been aquired from a real test. This would allow for engineers to vary parameters in the analysis such as speed of the CT, expected damping levels of the test article, and the amount of noise expected to be present in the channels. The importance of knowing the sensitivity to speed, damping, and noise will help guide decisions made by engineers acquiring data during the rollout event.

Running through the NASTRAN solution to generate time domain accelerations was a fairly straightforward task. However, prior to generating all this data, there were some basic checks that the analyst felt were important to perform to help anchor the approach. 
1. Correlation between the generated forcing functions are checked to see if the EMA MIMO approach to processing the data is valid.

2. Check the EMA MIMO post processing approach, using perfect broadband white noise signals, to verify all the target modes can be extracted.

3. Verify that the data being generated by NASTRAN using the generated forcing functions passes standard data quality checks performed on test data (i.e. the drive point Frequency Response Function (FRF) phase should not vary more than 180 degrees).

4. Compare FRFs generated using the perfect broadband white noise signals, FRFs generated using the GRFFs, and FRFs generated from MSC NASTRAN Solution 111 "Dynamic Frequency Domain Simulation" to ensure that all three sets of FRFs overlay on top of each other.

The plan was to treat the forcing functions as if they were shakers in a traditional EMA MIMO modal test. That is, each force direction at each truck produced its own uncorrelated broadband random signal that excites the structure. However, engineers already knew this not to be the case with the CT forcing functions [1]. The CT trucks produce harmonics due to shoe/roller interaction and other rotating equipment. In addition to the harmonics, the CT trucks are attached to each other via the CT chassis, thus no truck is truly independent from the other. This connection between the trucks will most likely cause the forces to be correlated to each other. It is not yet known exactly how much correlation between the truck forces will be present. Would there only be a limited amount of correlation between the forces and thus allow for modal extraction using the MIMO method? Does a certain speed show less correlation in the forces than another? These are questions that this analysis can answer assuming that the GRFFs are similar to the real forcing functions.

To determine if the GRFFs were sufficiently uncorrelated for a EMA MIMO approach to be used to extract modal parameters, the correlation coefficients between all 12 GRFFs for each speed analyzed was computed using the MATLAB function "corrcoef" and are shown in Figure 13.
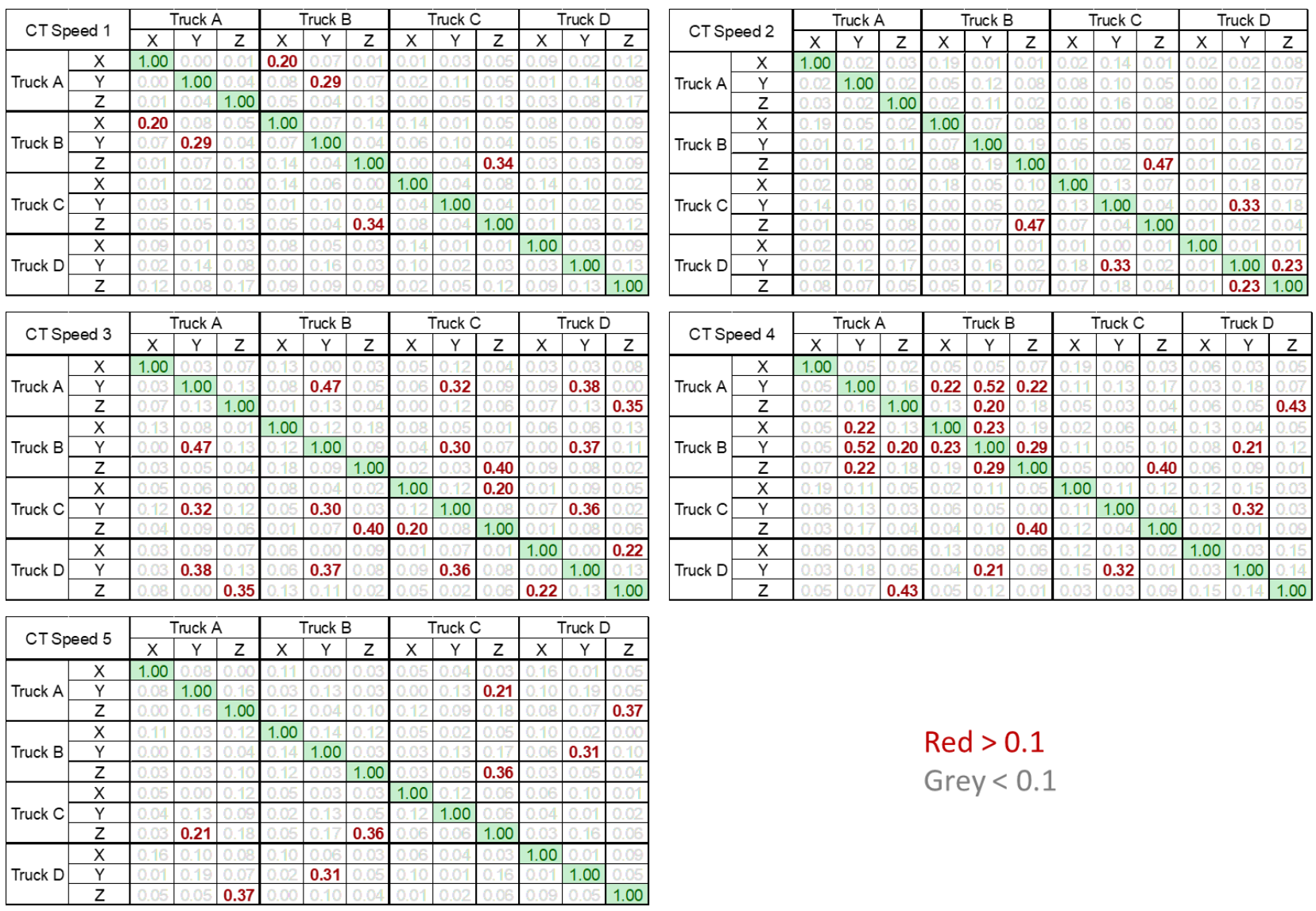

Red $>0.1$

Grey $<0.1$

Figure 13. Correlation Coefficients for Different CT Speeds 
Figure 13 shows CT speed has a significant impact on the amount of correlation between the GRFFs. Based on GRFFs correlation, the CT speeds 1,2, and 5 have the least correlation and appear to be best suited for an EMA MIMO approach for extracting modal parameters. CT speeds 3 and 4 have significantly higher correlation indicating an OMA MIMO approach may be better suited.

MSC NASTRAN SOL 112 modal transient solution was used to perform the force response analysis in this study. Eigenvalues were calculated up to twice the frequency range of interest for these analyses and residual vectors were always calculated. Critical damping of $1 \%$ was assigned to all eigenvalues and $100 \%$ was assigned to all residual vectors calculated in the analysis. To verify the MSC NASTRAN SOL 112 force response analysis was being used correctly, an analysis was performed with the 12 GRFFs replaced with 12 uncorrelated broadband white noise as the forcing functions. MATLAB was used to generate the 12 uncorrelated broadband Gaussian distributed white noise force time histories that were scaled with the same root mean square (RMS) levels as the GRFF's. Then ATA Engineering's MATLAB, Analysis and Test (IMAT) [5] software function "writefemap" was used to write these force time histories to a neutral file that in turn was read into the FEMAP software package. FEMAP was then used to generate the 12 load cards used in the MSC NASTRAN SOL 112 analysis. Figure 14 shows the correlation coefficients between the 12 uncorrelated broadband Gaussian distributed white noise force time histories.

\begin{tabular}{|c|c|c|c|c|c|c|c|c|c|c|c|c|c|}
\hline \multirow{2}{*}{\multicolumn{2}{|c|}{$\begin{array}{c}\text { Uncorrelated } \\
\text { Broadband Forcing Functions }\end{array}$}} & \multicolumn{3}{|c|}{ Truck A } & \multicolumn{3}{|c|}{ Truck B } & \multicolumn{3}{|c|}{ Truck C } & \multicolumn{3}{|c|}{ Truck D } \\
\hline & & $\mathrm{X}$ & $\mathrm{Y}$ & Z & $\mathrm{X}$ & $\mathrm{Y}$ & Z & $\mathrm{X}$ & $\mathrm{Y}$ & Z & $X$ & $\mathrm{Y}$ & Z \\
\hline \multirow{3}{*}{ Truck A } & $\mathrm{X}$ & 1.00 & 0.00 & 0.00 & 0.00 & 0.00 & 0.00 & 0.00 & 0.00 & 0.00 & 0.00 & 0.00 & $\overline{0.00}$ \\
\hline & $\mathrm{Y}$ & 0.00 & 1.00 & 0.00 & 0.00 & 0.00 & 0.00 & 0.00 & 0.00 & 0.00 & 0.00 & 0.00 & 0.00 \\
\hline & Z & 0.00 & 0.00 & 1.00 & 0.00 & 0.00 & 0.00 & 0.00 & 0.00 & 0.00 & 0.00 & 0.00 & 0.00 \\
\hline \multirow{3}{*}{ Truck B } & $\bar{x}$ & $\overline{0.00}$ & $\overline{0.00}$ & 0.00 & 1.00 & 0,00 & $\overline{0.00}$ & $\overline{0.00}$ & 0.00 & 0.00 & 0.00 & 0.00 & 0.00 \\
\hline & $\bar{Y}$ & 0.00 & 0.00 & 0.00 & 0.00 & 1.00 & $\overline{0.00}$ & 0.00 & 0.00 & 0.00 & 0.00 & 0.00 & 0.00 \\
\hline & Z & 0.00 & 0.00 & 0.00 & 0.00 & 0.00 & 1.00 & 0.00 & 0.00 & 0.00 & 0.00 & 0.00 & 0.00 \\
\hline \multirow{3}{*}{ Truck C } & $\bar{X}$ & O & 0.00 & 0.00 & 0.00 & 0.00 & 0.06 & 1.00 & 0.00 & 0.00 & 0.00 & 0.00 & 0.00 \\
\hline & $\mathrm{Y}$ & 0.00 & 0.00 & 0.00 & 0.00 & 0.00 & 0.00 & 0.00 & 1.00 & 0.00 & 0.00 & 0.00 & 0.00 \\
\hline & Z & 0.00 & 0.00 & 0.00 & 0.00 & 0.00 & 0.00 & 0.00 & 0.00 & 1.00 & 0.00 & 0.00 & 0.00 \\
\hline \multirow{3}{*}{ Truck D } & $\mathrm{X}$ & 0.00 & 0.00 & 0.00 & 0.00 & 0.00 & 0.00 & 0.00 & 0.00 & 0.00 & 1.00 & 0.00 & 0.00 \\
\hline & $\mathrm{Y}$ & 0.00 & 0.00 & 0.0 & 0.00 & 0.00 & 0.00 & 0.00 & 0.00 & 0.00 & 0.00 & 1.00 & 0.0 \\
\hline & Z & 0.00 & 0.00 & 0.00 & 0.00 & 0.00 & 0.00 & 0.00 & 0.00 & 0.00 & 0.00 & 0.00 & 1.00 \\
\hline
\end{tabular}

Figure 14. Uncorrelated Broadband Forcing Function Correlation Coefficients

The acceleration response time histories underwent standard time-domain and had Frequency Response Functions (FRFs) computed on them, which in turn underwent standard frequency-domain data quality checks (e.g. drive point FRF phase should not vary more than 180 degrees, etc.,). Standard frequency-domain data quality checks were peformed on the SOL 112 FRF, of which one is shown in Figure 15. The FRFs were generated using the same process that would be utilized when the GRFF's are applied (see Section 0).

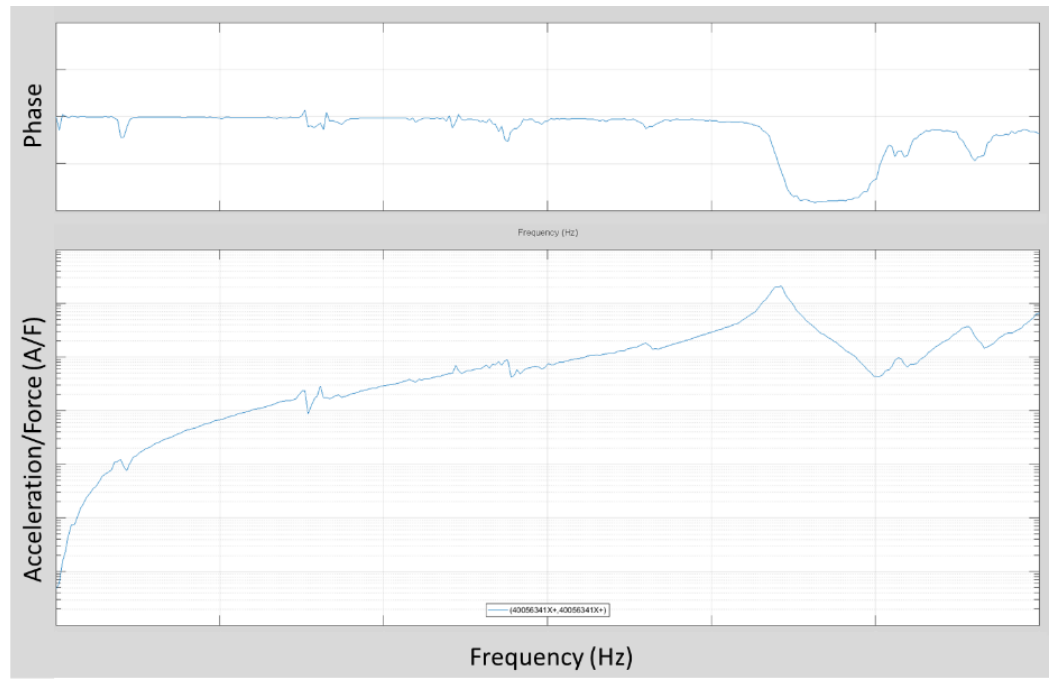

Figure 15. Drive-Point FRF Check Showing No Phase Shifting Outside 180 Degrees 
These FRFs were very clean and all modal parameters were successfully extracted (Figure 16) and agreed very well with the FEM modal parameters. This was the expected outcome because everything is idealized (i.e. no correlated forces, no noise, etc.). This was a great way to confirm there were no unit problems or other similar user issues that could potentially cause inaccuracies in the approach.
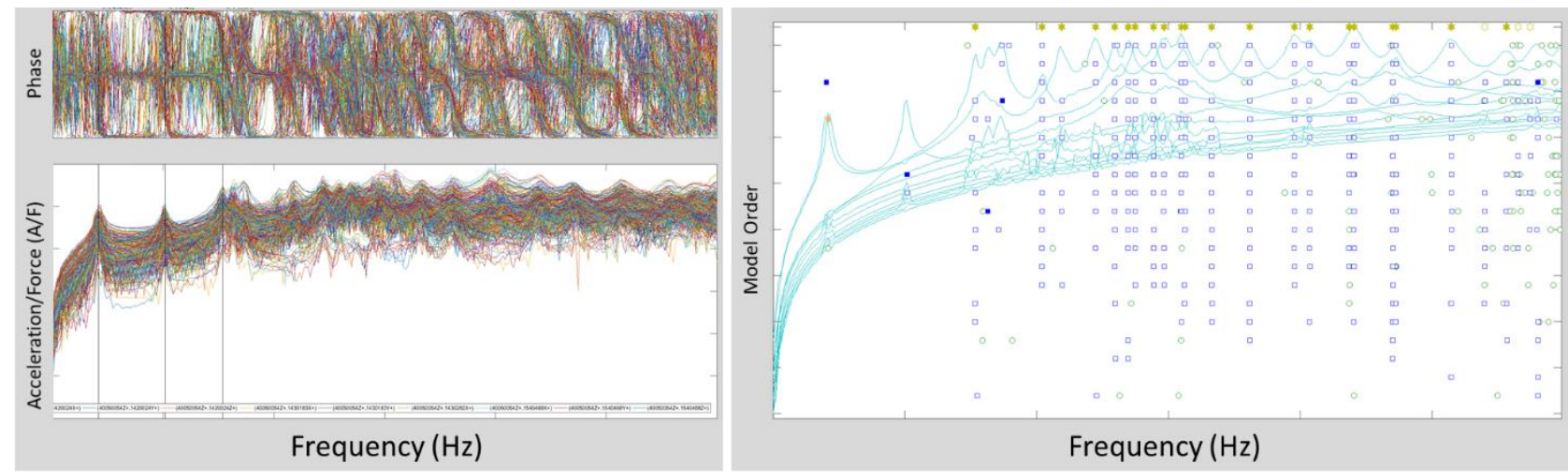

Figure 16. FRF and Stability Diagram using Uncorrelated Broadband Forces

For the final data quality check, the FRFs that were generated by applying the GRFF's were compared to the FRFs generated by applying perfect uncorrelated broadband white noise force time histories and FRF computed from acceleration response time histories generated using MSC NASTRAN SOL 111 modal frequency solution sequence where the input PSD's were those corresponding to the uncorrelated broadband white noise forcing functions used in the SOL 111 analysis. The FRFs matched very well for the first half of the frequency range of interest, but above that the SOL 111 FRFs started to diverge as shown in Figure 17.

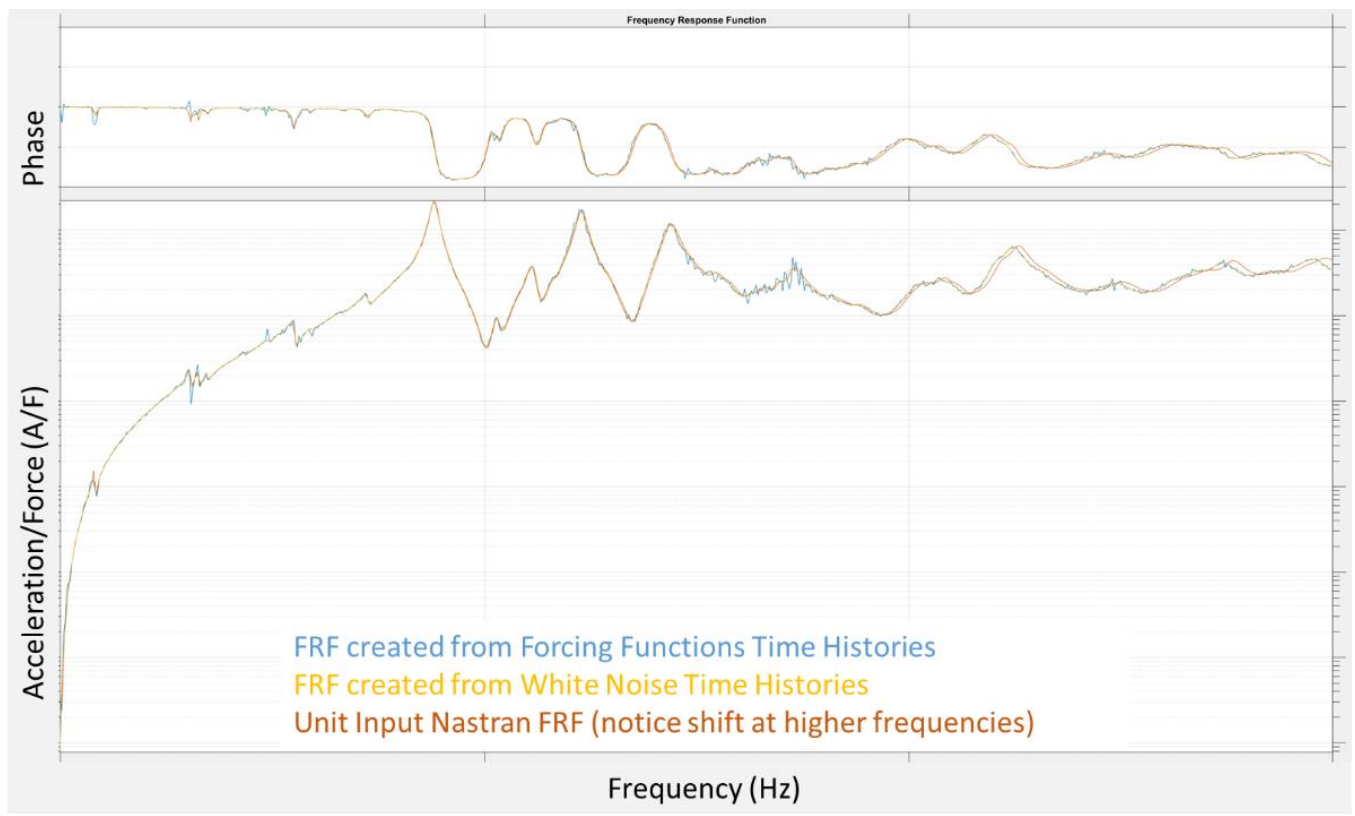

Figure 17. Analytical FRF Overlay Check

The SOL 111 FRF exhibited a frequency shift, which grew steadily as the frequency increased. The following three potential sources of error were looked at:

1. Round off error in the time domain data.

2. Restarting the MSC NASTRAN decks without recalculating the eigenvalues every time.

3. Differences in the residual vectors being calculated between the SOL 111 and SOL112 solutions. 
First, if the issue was round off error, was investigated by lowering the sample frequency of the uncorrelated broad band forces being generated, which resulted in the SOL 111 FRF (orange curve) starting to diverge at a low frequency, as shown in Figure 18. It should be noted that large field format was utilized to minimize round off error.

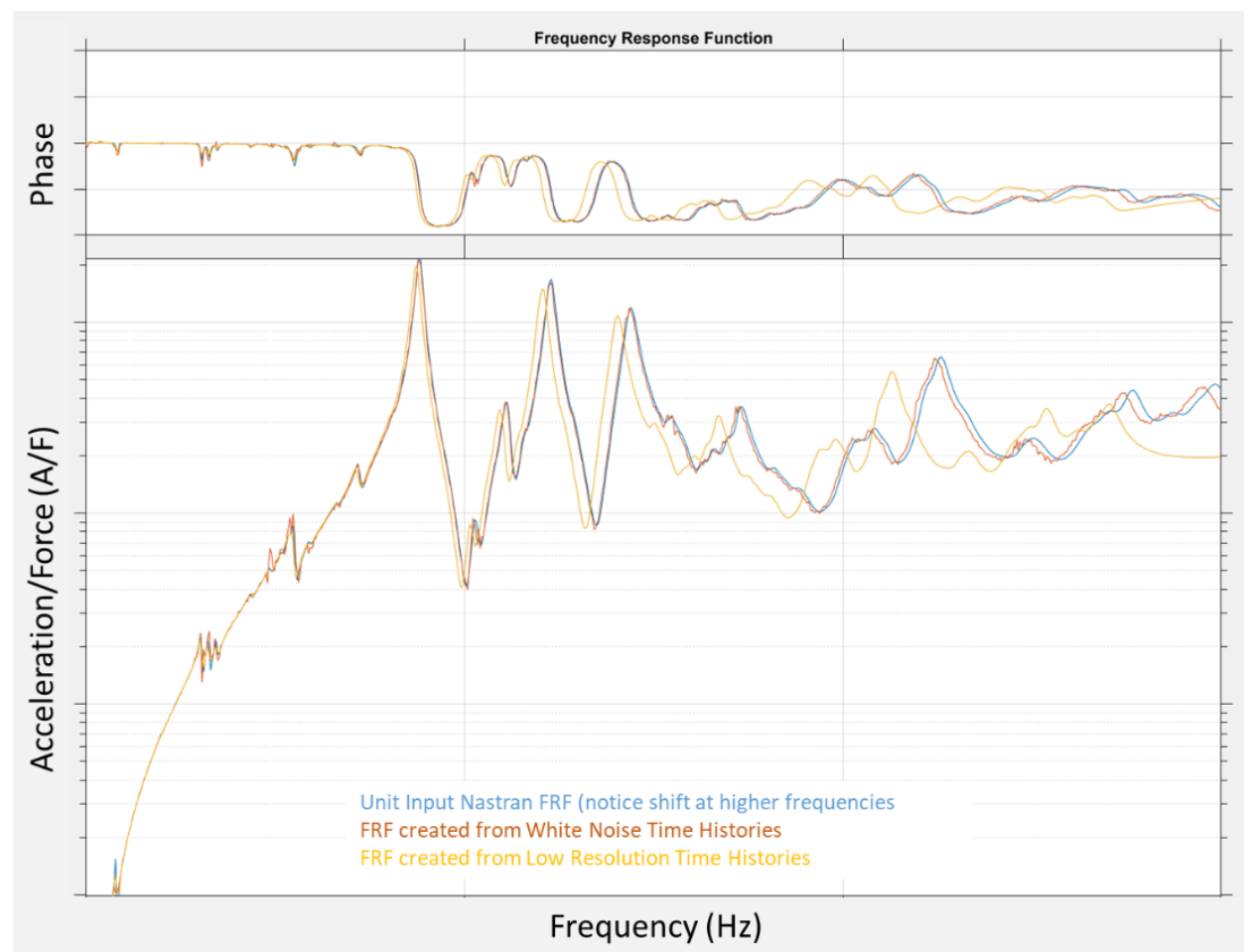

Figure 18. Effect of Reduced Resolution in Uncorrelated Broadband Forces

Next, could the issue be due to not setting up the NASTRAN restart decks correctly? A solution was run without any restarts and compared back to the original. No improvement on the results were seen.

Finally, could the issue lie with the fact that the residual vectors being used in both solutions be different from each other? This was verified not to be the case by purposefully not calculating as many residual vectors on one analytical case and seeing the effect to the data. The resulted in slight in a very slight change magnitudes, but not frequencies, as shown in Figure 19.

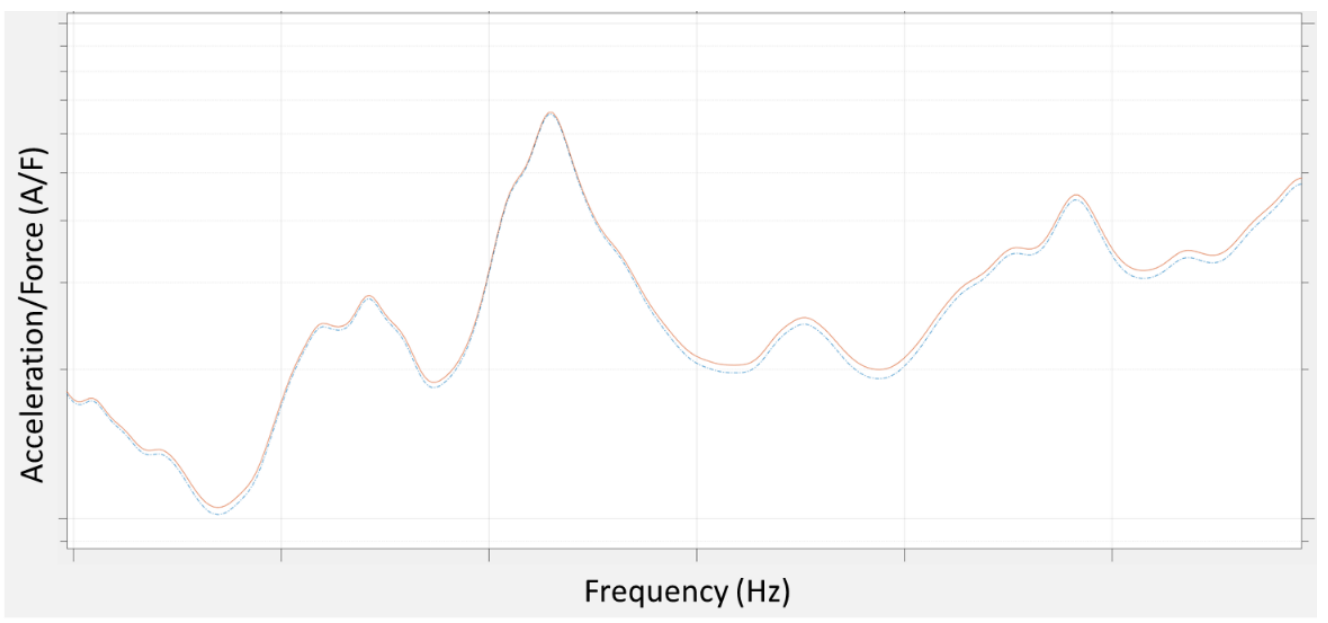

Figure 19. Difference in Residual Vectors Impact on the FRF 
After all these checks, it still remains an uncertainty as to why the results between the SOL 112 time domain solution and the SOL 111 frequency domain solutions slowly diverge from each other. It is suspected that this frequency divergence is tied to the resolution of the time data in some way.

This FRF frequency divergence effect was avoided in this study by including elastic body modes up to double the frequency range of interest, which was already being done based on best practices. Hence the use of MSC NASTRAN SOL 112 in this study has been verified and the noise free acceleration response time histories valid.

\subsection{Force Response Analysis Results}

A total of 12 forces ( 3 orthogonal translational forces at 4 CT trucks) at the locations discussed previously in Section 3.1 of this paper. The application of these forces was carried out using MSC NASTRAN's Solution 112, "Dynamic Time Domain Simulation tool." The forcing functions were different depending on the speed of the CT. The analyst was supplied five sets of 12 forcing functions. In additon to having 5 different speeds to vary, 3 different damping levels (1\%, 3\%, and 5\% critical damping) and 3 different sensor noise levels (30 $\mu \mathrm{grms}, 100 \mu \mathrm{grms}$, and $200 \mu \mathrm{grms}$ ) were additionally considered as parameters in the study. The modal damping levels of $1 \%, 3 \%$, and $5 \%$ were believed to envelope the actual modal damping levels based upon modal testing experience. The sensor/ambient background noise levels of $30 \mu$ grms was based upon the broadband resolution of the accelerometer that was selected at the time to collect the data. The higher sensor/ambient background noise levels of $100 \mu \mathrm{grms}$ and $200 \mu \mathrm{grms}$ were included as medium and high level background noise levels. Actual sensor noise levels and ambient background noise levels were not available for this study.

The analytical results of the NASTRAN time domain dynamic simulation were brought into IMAT [5], where its Signal Processing toolbox was used to of IMAT, the time domain accelerations and forces were processed into FRFs.

In order to determine what references to use when generating the FRFs, many different subsets of the GRFFs were tried, guided by their correlation results shown in Figure 13, to determine which subset produced the cleanest FRF. Figure 20 shows a comparison of the FRFs generated using all 12 GRFFs as references and using a smaller subset of GRFFs as references. In the case shown, the subset of GRFFs used as references was chosen to minimize the correlation between these forces as much as possible. It became obvious during this study that using subsets of the 12 GRFFs as references does not to produce as clean of FRFs as using all GRFFs as references. Based on this finding, all the modal parameter extractions were performed using acceleration response time histories generated utilizing all 12 GRFFs as references.
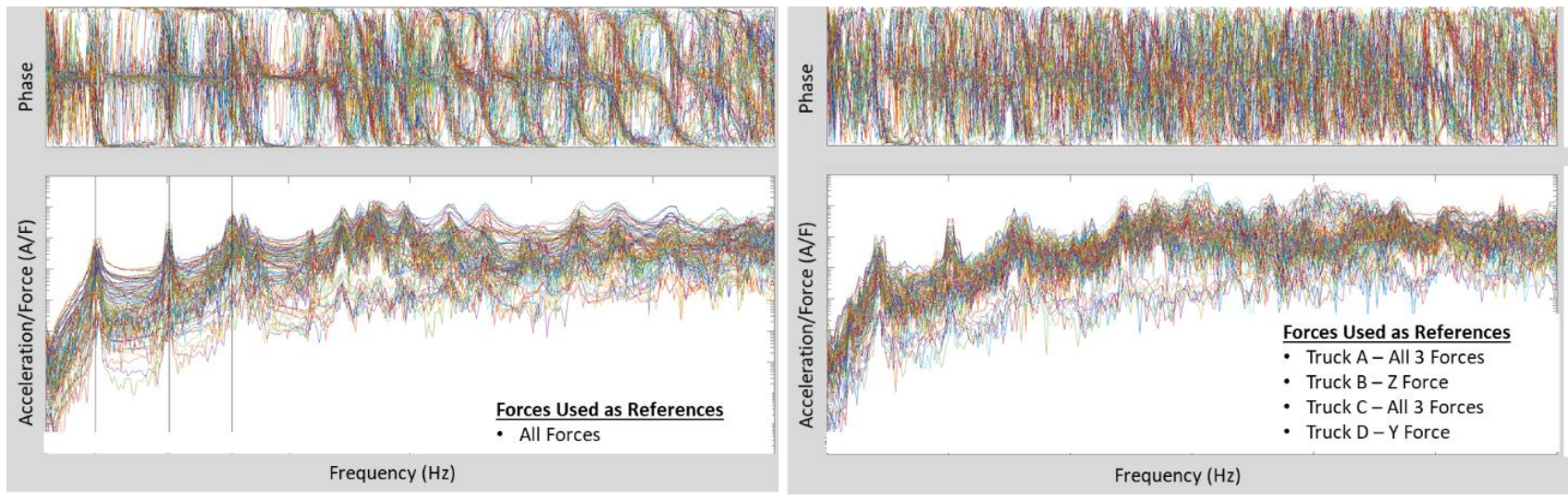

\section{Figure 20. FRFs using All Forces as References vs FRFs using Subset of Least Correlated Forces as References, CT Speed 2}

IMAT's AFPOLY toolbox was used to peform the modal extaction with the complex mode indicator function (CMIF) utilized to help select pole estimates. It was understood that the answers to exactly what the modal frequencies and damping values should be were already available because they were the FEM results in this study. However, in an effort to be as "realistic as possible", only pole estimates that were able to be automatically selected by the IMAT AFPOLY software or that corresponded to a very clear peak in the CMIF were considered in each modal extraction.

The parameter space performed in this studied (5 speed settings, 3 different damping values, 3 noise levels) is summarized in Table 1. All five CT speeds were analyzed with 1\%, 3\% and 5\% modal damping, but no sensor/ambient background noise. 
Then the CT speed 2 was analyzed with a modal damping level of $1 \%$ with the $30 \mu$ grms, $100 \mu$ grms, and $200 \mu$ grms sensor/ambient background noise levels.

Table 1. Summary of Parameter Variation

\begin{tabular}{|c|c|c|c|c|c|}
\hline \multicolumn{7}{|c|}{ Parameter Variation Summary } \\
\hline & \multicolumn{7}{|c|}{ CT Speed } \\
\hline Damping & $\mathbf{1}$ & $\mathbf{2}$ & $\mathbf{3}$ & $\mathbf{4}$ & $\mathbf{5}$ \\
\hline $\mathbf{1 \%}$ & $\mathrm{X}$ & $\mathrm{X}$ & $\mathrm{X}$ & $\mathrm{X}$ & $\mathrm{X}$ \\
\hline $\mathbf{3 \%}$ & $\mathrm{X}$ & $\mathrm{X}$ & $\mathrm{X}$ & $\mathrm{X}$ & $\mathrm{X}$ \\
\hline $\mathbf{5 \%}$ & $\mathrm{X}$ & $\mathrm{X}$ & $\mathrm{X}$ & $\mathrm{X}$ & $\mathrm{X}$ \\
\hline & \multicolumn{5}{|c|}{ CT Speed } \\
\hline $\begin{array}{c}\text { Broadband Noise } \\
\text { (1\% Damping) }\end{array}$ & $\mathbf{1}$ & $\mathbf{2}$ & $\mathbf{3}$ & $\mathbf{4}$ & $\mathbf{5}$ \\
\hline 30 ugrms & & $\mathrm{X}$ & & & \\
\hline 100 ugrms & & $\mathrm{X}$ & & & \\
\hline 200 ugrms & & $\mathrm{X}$ & & & \\
\hline
\end{tabular}

Table 2 shows the percentage of the 28 target modes in this study that were automatically or very clearly selected from a stability diagram/CMIF. With no sensor/ambient background noise present, the highest number of target modes could be identified for $\mathrm{CT}$ speed 2. With no sensor/ambient background noise present, the first three target modes, which were the most challenging modes to extract cleanly in all data sets, were most easily identified for CT speed 5.

As expected, increasing modal damping and the sensor/ambient background noise levels make identification of the target modes more difficult. In the case of sensor/ambient background noise however, the addition of any level significantly reduces the ability to identify target modes. This study did not run the combined highest levels of noise and highest levels of damping in the same case. This was primarily because it was perceived by the analyst that this would generate the unrealistic case where no target modes could be extracted. In reality, the noise levels and damping levels vary from frequency to frequency and as such, it is likely that some modes will not be extracted because of a high of combination of noise level and damping while others will be extracted. Detailed accounts of all the results are presented in the upcoming paragraphs.

Table 2. Percentage of Target Modes Extracted

\begin{tabular}{|c|c|c|c|c|c|}
\hline \multicolumn{6}{|c|}{ Percentage of Target Modes Extracted } \\
\hline & $\mathbf{1}$ & $\mathbf{2}$ & $\mathbf{3}$ & $\mathbf{4}$ & $\mathbf{5}$ \\
\hline Damping & $\mathbf{1} \%$ & $93 \%$ & $93 \%$ & $89 \%$ & $93 \%$ \\
\hline $\mathbf{1 \%}$ & $75 \%$ & $89 \%$ & $75 \%$ & $79 \%$ & $82 \%$ \\
\hline $\mathbf{3} \%$ & $36 \%$ & $46 \%$ & $43 \%$ & $36 \%$ & $39 \%$ \\
\hline $\mathbf{5 \%}$ & \multicolumn{5}{|c|}{ CT Speed } \\
\hline $\begin{array}{c}\text { Broadband Noise } \\
\text { (1\% Damping) }\end{array}$ & $\mathbf{1}$ & $\mathbf{2}$ & $\mathbf{3}$ & $\mathbf{4}$ & $\mathbf{5}$ \\
\hline $\mathbf{3 0}$ ugrms & & $61 \%$ & & & \\
\hline $\mathbf{1 0 0}$ ugrms & & $43 \%$ & & & \\
\hline $\mathbf{2 0 0}$ ugrms & & $43 \%$ & & & \\
\hline \multicolumn{7}{|c|}{} \\
> 75\% Target Mode Extraction \\
> 50\% Target Mode Extraction \\
$<50 \%$ Target Mode Extraction
\end{tabular}

\subsubsection{Speed Parameter Sensitivity}

Varying the CT speed did not have a significant impact on the overall ability to extract modal parameters in this simulation. CT speed variations primarily impacted identification of the first three target modes. In the stability diagrams shown in Figure 21 for CT Speed 1 and CT Speed 5 with 1\% modal damping level and no sensor/ambient background noise, there is almost no difference in the quality of the CMIF or race track pattern of the pole estimates, except for the first three target modes. CT speed 5 with no sensor/ambient background noise was the only CT speed where the first three modes were cleanly extracted from the simulation. This can be more clearly observed in the cross-orthogonality matrices that were generated for each CT speed and shown in Figure 22. 


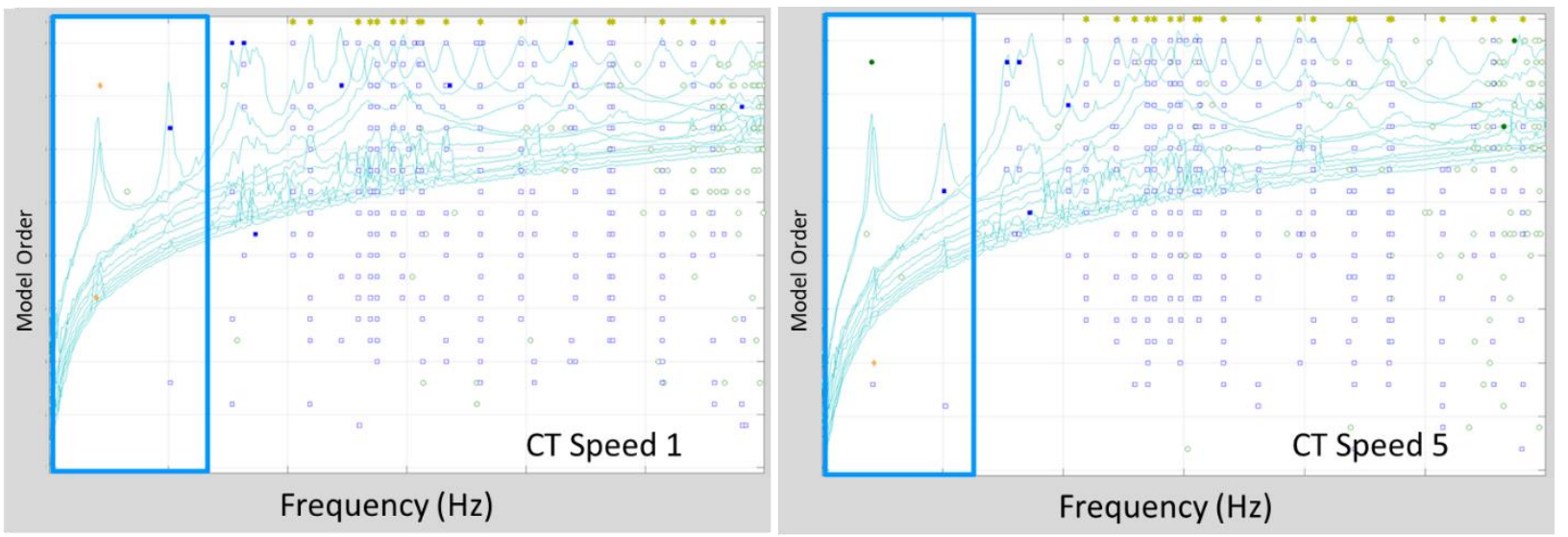

Figure 21. Stability Diagrams for the CT Speed 1 (left) and CT Speed 5 (right), 1\% Modal Damping Level, No Sensor/Ambient Background Noise

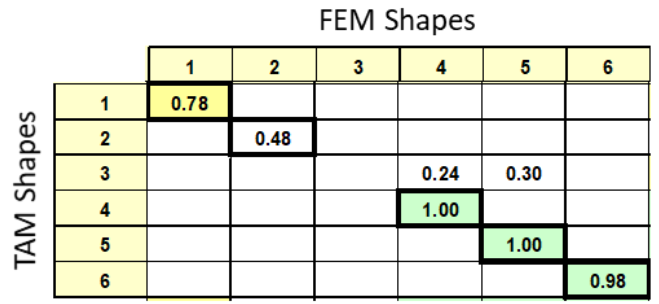

CT Speed 1

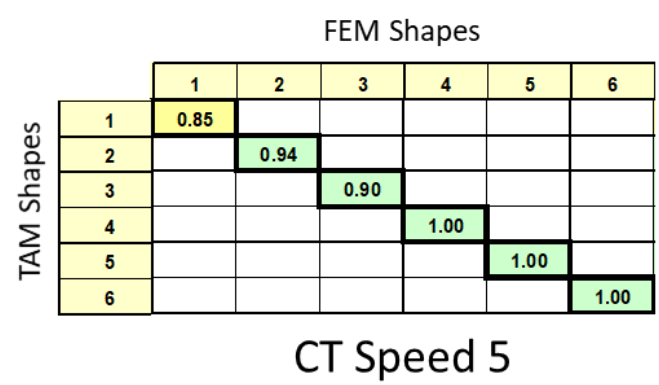

CT Speed 5

Figure 22. Cross Orthogonality Matrices of the Lowest Frequency Modes for CT Speed 1 (left) and CT Speed 5 (right), 1\% Modal Damping And No Sensor/Ambient Background Noise

\subsubsection{Damping Parameter Sensitivity}

This study showed that, with no sensor/ambient background noise present, increasing levels of modal damping made identification of closely spaced target modes more difficult. Modal damping levels exceeding 3\% make it extremely difficult to identify the target modes and if this is the case during actual testing, the test engineers would probably not be able to identify more than the first few target modes. In Figure 23, the gold stars at the top of each stability matrix/CMIF overlay indicate what pole estimates were able to be automatically selected by the AFPOLY software. In this study, these automatically selected pole estimates were used as an indicator of how strong a mode was excited and how easy it would be to identify it. Pole estimates not automatically selected in this study indicate modes the test engineers most likely will have a very challenging time trying to identify.

In an attempt to keep the study as "realistic" as possible, no manually selected poles were used even if they could be accurately selected from the stability diagram. This precluded the analyst from selecting poles based upon knowledge of the FEM modes. Figure 24 shows the cross-orthogonality matrices of these automatically selected modes for each level of modal damping considered at a CT speed 2 (the speed most likely to provide clean modes). When the modal damping level increased above $3 \%$, the software's ability to select pole estimates was cut nearly in half and only 13 of the 28 target modes were able to be extracted.

One part of this simulation that did not hold true to what is typically seen in a real test environment is that increasing the damping from $1 \%$ to $3 \%$ actually helped the modal extraction of the first three target modes. Extracting the first three target modes in this study was constant challenge and this inconsistency is most likely due to the exact pole estimate chosen. This finding would not be observed in the actual test environment. 


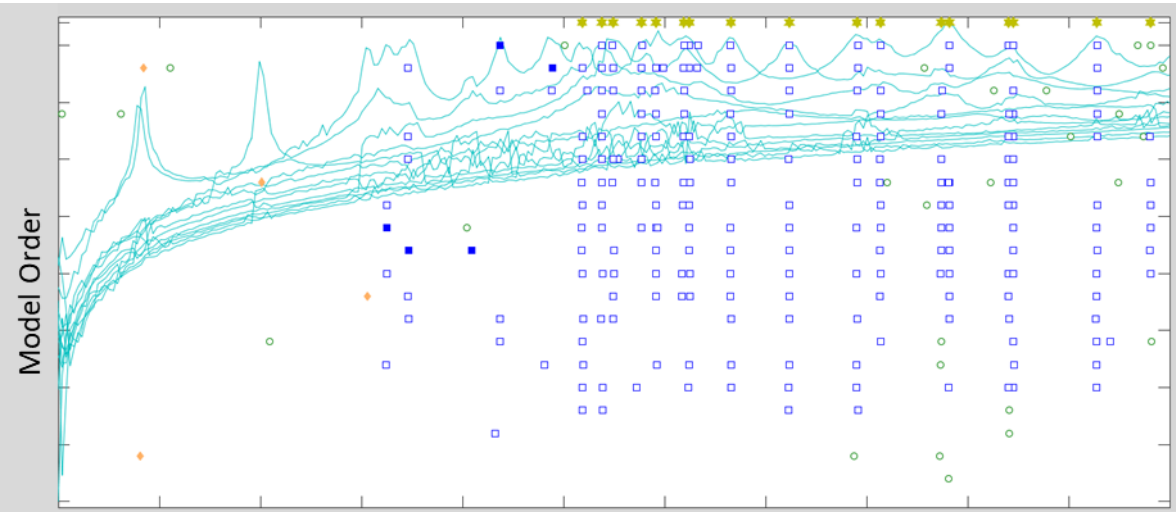

Frequency $(\mathrm{Hz})$

1\% Damping - CT Speed 2

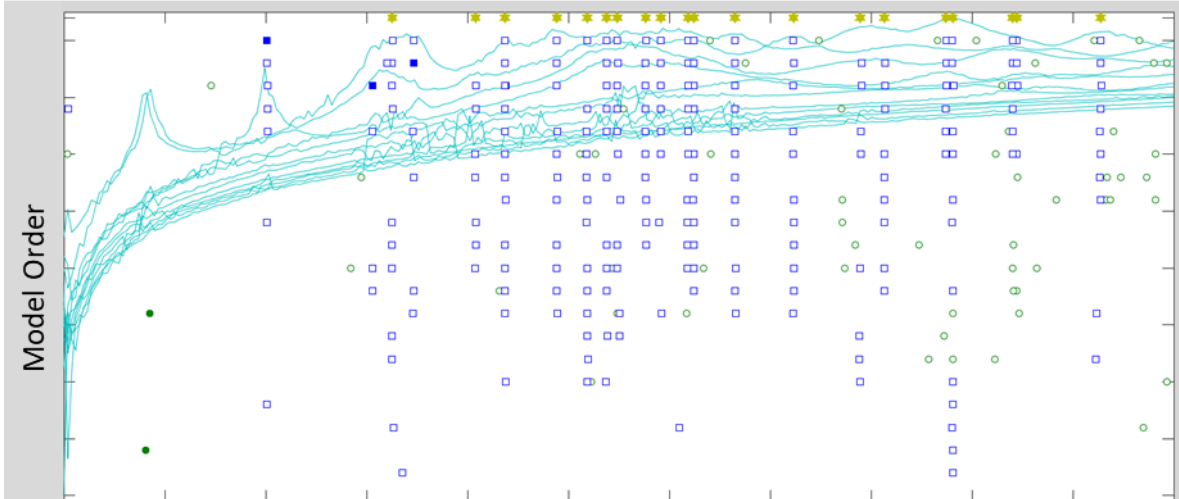

Frequency $(\mathrm{Hz})$

3\% Damping - CT Speed 2

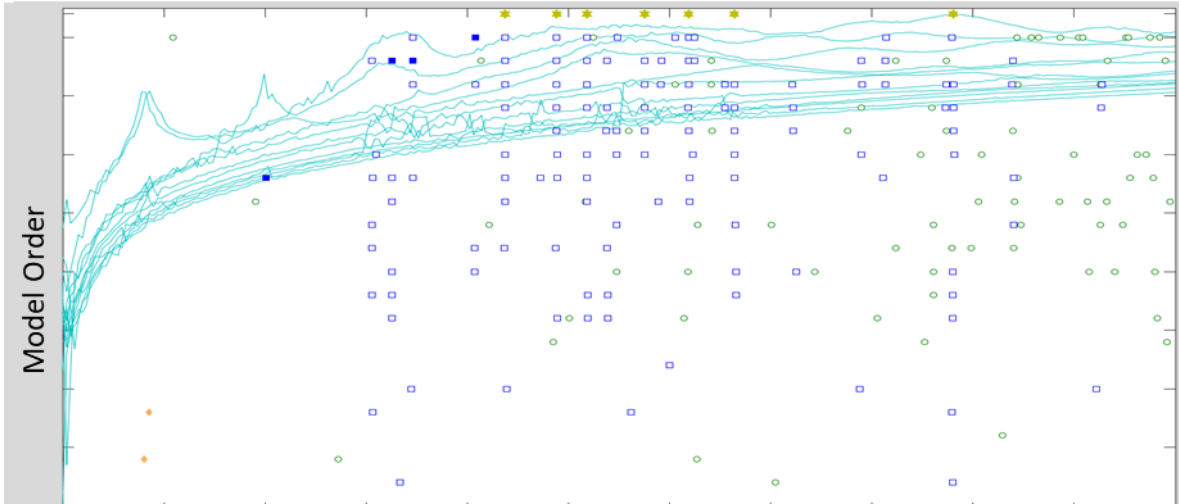

Frequency $(\mathrm{Hz})$

5\% Damping - CT Speed 2

Figure 23. Stability Diagrams for Varying Modal Damping Levels with CT Speed 2, No Sensor/Ambient Background Noise 


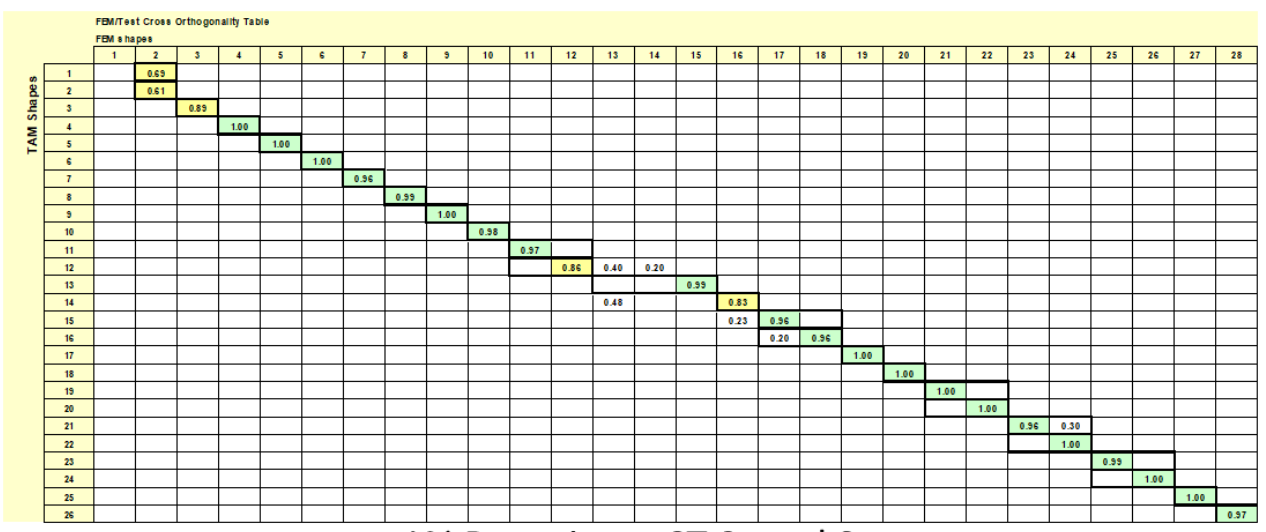

1\% Damping - CT Speed 2

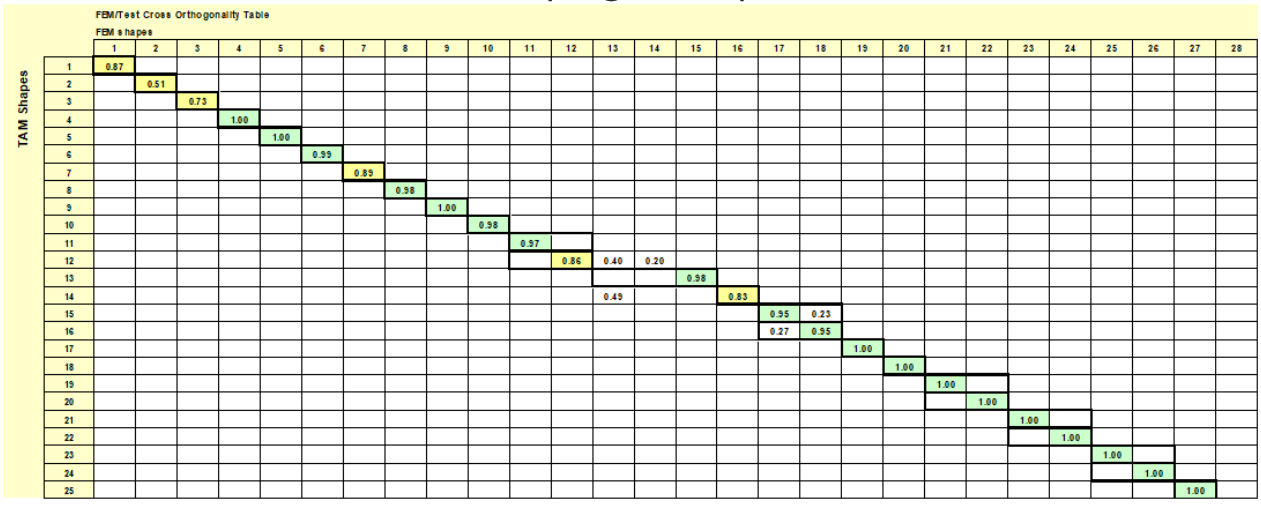

3\% Damping - CT Speed 2

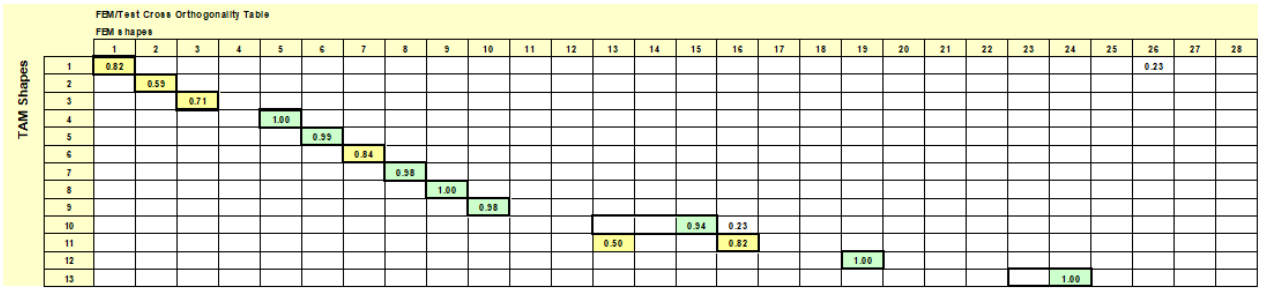

5\% Damping - CT Speed 2

\section{Figure 24. Cross Orthogonality Matrices for Varying Damping Levels with a CT Speed 2, No Sensor/Ambient Background Noise}

\subsubsection{Broadband Noise Parameter Sensitivity}

Finally, the last parameter that was varied was the amount of ambient noise that might be present in the accelerometer measurements. Broadband noise was not applied to the forces in this study due to the fact that accelerometers usually have a significantly higher noise levels than force measurements do. Three different levels of broadband noise were utilized in this study. A broadband noise signal was generated and summed into each of the individual SDAS/CTDAS accelerations. The data was processed the same way as it was in the damping parameter sensitivity study. In an attempt to save time, the noise was only applied to CT speed 2 and a damping value of $1 \%$. This choice was made because CT speed 2 was deemed the best overall modal extraction speed based on the results of this analysis. The results were impacted in a similar way to increasing the overall damping level, but the effect is more significant. Simply increasing the noise to the minimum of 30 $\mu$ grms reduced the selected modes from 26 to 17 . If there is much more than $30 \mu$ grms present in each accelerometer signal, the ability for the test engineers to extract target modes becomes a very difficult task. The reader can observe in Figure 25 and Figure 26 that the selection of modes using automated tools was significantly impacted when the noise level was increased from $30 \mu$ grms to $100 \mu \mathrm{grms}$. Once that $100 \mu \mathrm{grms}$ threshold was hit however, it did not seem to reduce the number of modes that could be selected when the noise was then again increased to $200 \mu$ grms. This was an unexpected occurrence and something that is not likely to hold true in the real test. 


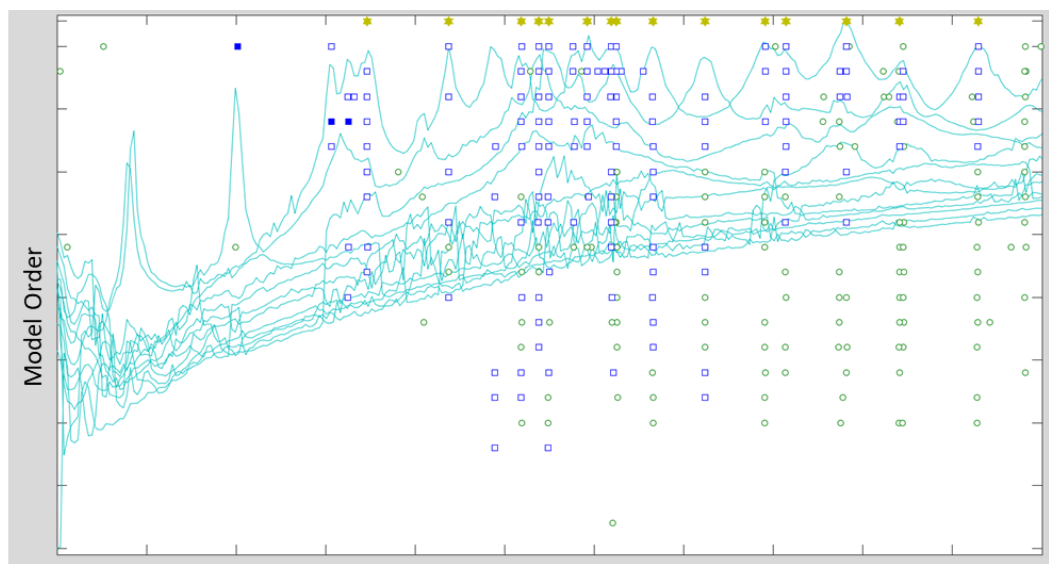

Frequency $(\mathrm{Hz})$

30 ugrms Ambient Noise, 1\% Damping, CT Speed 2

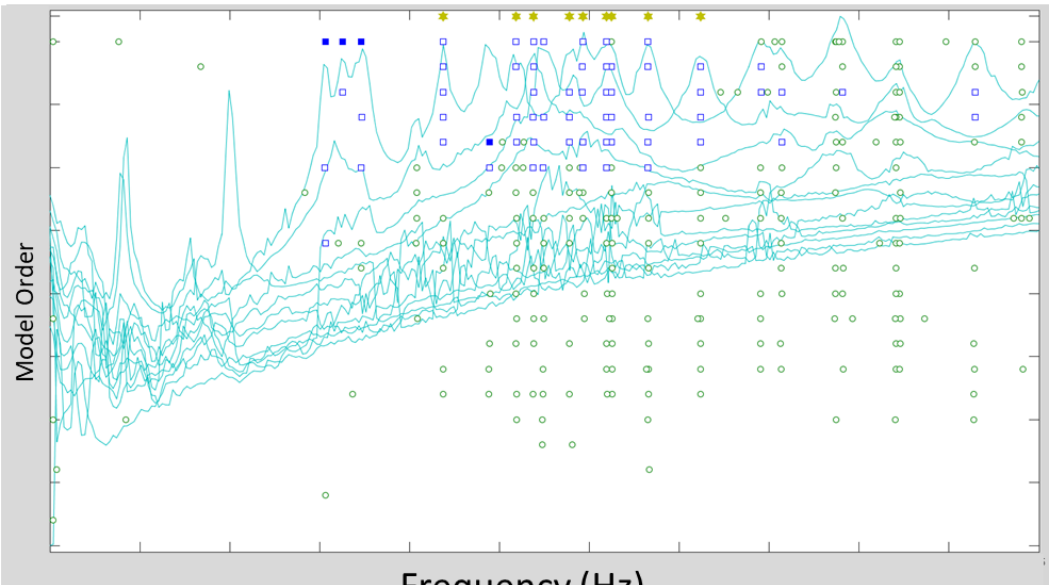

Frequency $(\mathrm{Hz})$

$100 \mu$ grms Ambient Noise, 1\% Damping, CT Speed 2

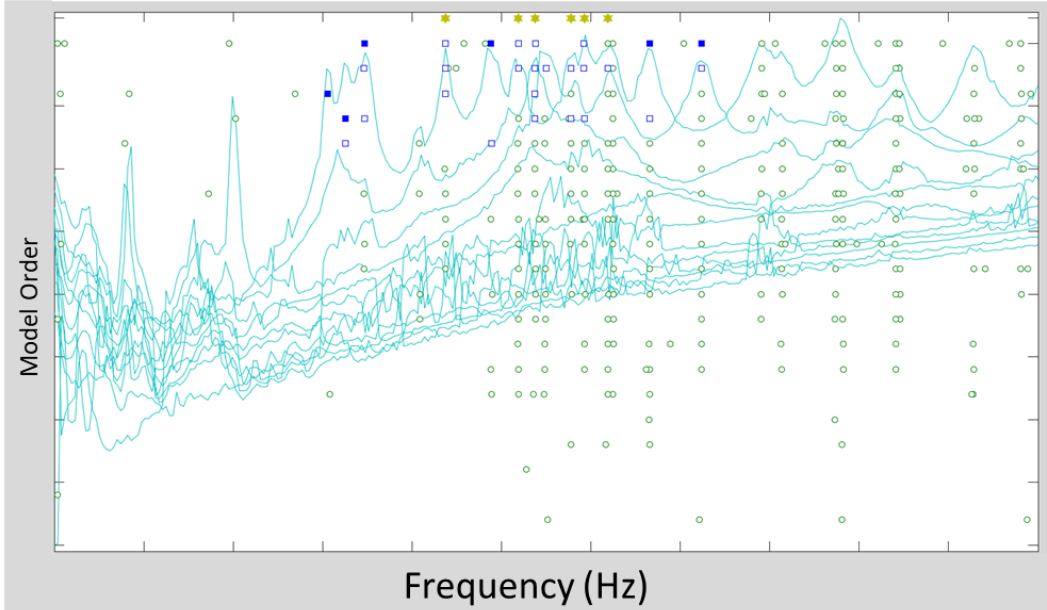

$200 \mu$ grms Ambient Noise, 1\% Damping, CT Speed 2

Figure 25. Stability Diagrams from Varying Broadband Noise Levels with CT Speed 2 and 1\% Damping 


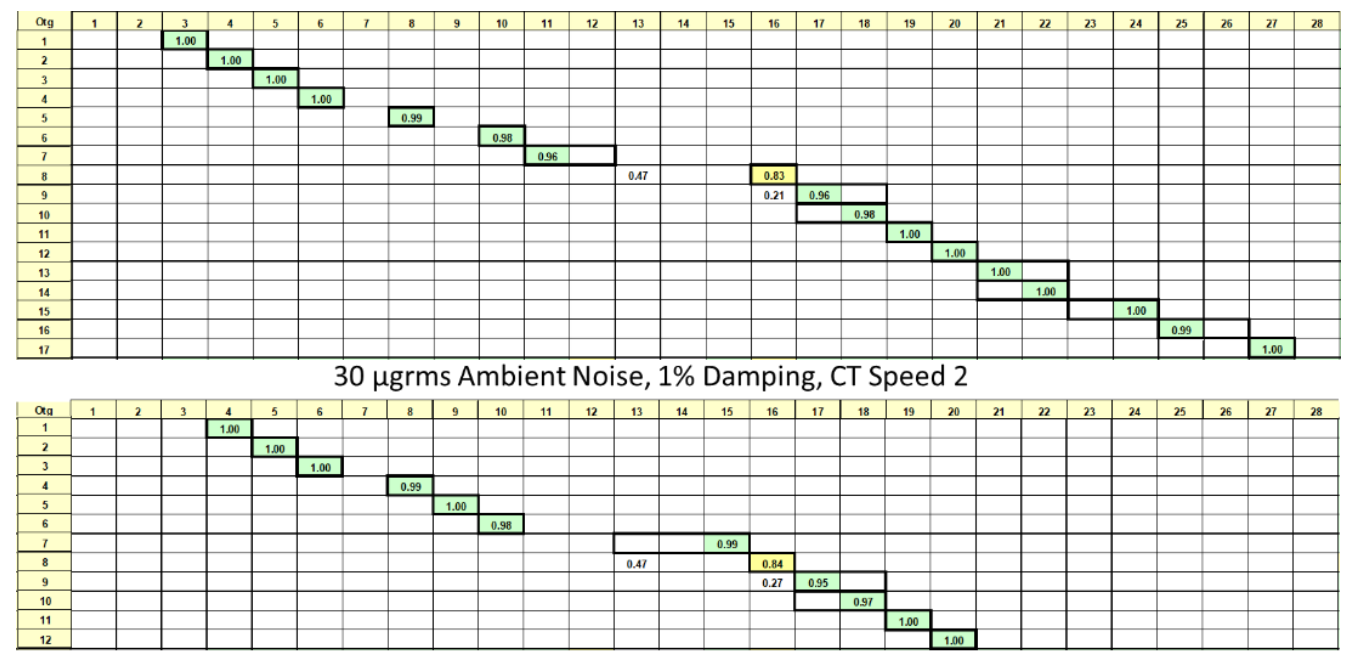

$100 \mu$ grms Ambient Noise, 1\% Damping, CT Speed 2

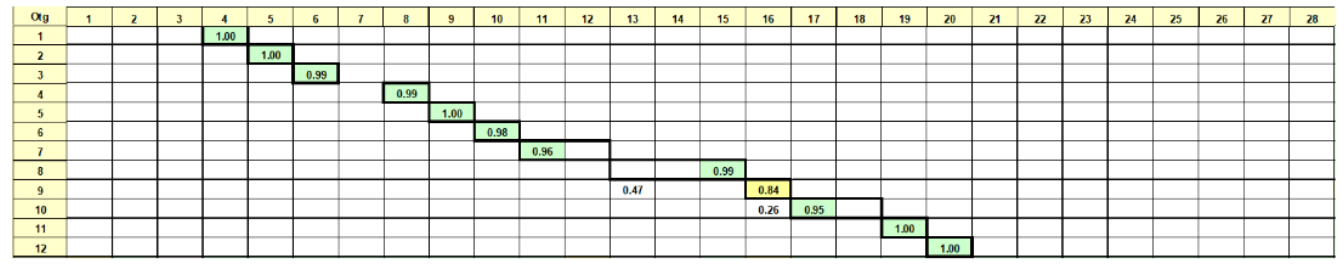

$200 \mu$ grms Ambient Noise, 1\% Damping, CT Speed 2

Figure 26. Cross Orthogonality for Varying Broadband Noise Levels with CT Speed 2 and 1\% Damping

\subsection{SUMMARY}

All cases studied (5 speed settings, 3 different damping values, 3 noise levels) showed an ability to extract a subset of the 28 identified target modes. With that said however, it is important to understand that specific values of certain parameters can help the test engineers obtain the best chances of success. The speed of the CT does not show a significant sensitivity in degrading the ability to extract the target modes. CT speed 2 showed the best overall potential for extracting the largest percentage of the target modes while a CT speed 5 showed the best potential for extracting the first three modes. These first three modes were the most challenging modes to extract cleanly in all data sets no matter what the parameters were set to. Increasing the damping of the modes always shows a tendency to make modal extraction more difficult. In this case, there is a significant degradation of the extraction ability when the damping values grow above $3 \%$. Finally, the ambient noise level present in this simulation proved to be a highly sensitive variable that could make or break the ability to extract a significant majority of the identified target modes. The addition of a nominal amount of ambient noise to the analysis showed a 30\% drop in extracted modes. Increasing the ambient noise level much more than a nominal amount lowered the extracted modes by an additional $20 \%$.

The major assumptions that this analytical work relies heavily upon is that the ML and CT FEM's both behave dynamically similar to the actual as-built hardware and the GRFFs are similar to the real forcing functions of a rollout event. It is understood by the author that this is rarely the case without some correlation having been done previously between the test data and analytical models. It is also understood that test data is always more challenging to extract modes from than the "perfect" analytical data simulated in models. The analysts' way of limiting this effect was to utilize the software to select the majority of the modes and having that act as the benchmark of whether a mode could be extracted in test data or not. It is the author's opinion that limiting the selection of target modes to those selected by the software is probably overconservative. Experience has shown that an experienced test engineer can usually select more modes that the software will.

The reader should not take these results to mean that this author is advocating for the elimination of a fullly instrumented traditonal modal test in leiu of using a rollout event to extract modal parameters for FEM correlation. The primary purpose of this analysis is to inform the reader of an opportunity to aquire supplemental modal test data that provides the ability to verify and validate the FEM under different load conditions and different ML tower configurations than currently planned in traditional modal test configurations. 


\subsection{REFERENCES}

[1] "Development of Generic Crawler/Transporter Rollout Forcing Functions for Coupled System Dynamics Analysis," NASA Exploration Systems Directorate/Cross-Program Systems Integration Technical Assessment Report, ESD 20038, July 31, 2018.

[2] "Integrated Test Requirements for Rollout Fatigue Loads Spectra Methodology Verification with Flight Configuration," NSTS 60539 (retired), September 24, 2004.

[3] "Crawler Transporter (CT)/MLP System Overview / Structural Models in Support of Roll-out Fatigue Spectra Analysis," United Space Alliance, October 21, 2003.

[4] "Loads Analysis of Spacecraft and Payloads", NASA-STD-5002, June 21, 1996.

[5] Interface between MATLAB, Analysis, and Test (IMAT) Version 7.2.0 build 4088 09/28/2018 Software by ATA engineering. 